\title{
Soft structures of groups and rings
}

\author{
Jayanta Ghosh ${ }^{1}$, Dhananjoy Mandal ${ }^{2}$, T. K. Samanta ${ }^{3}$ \\ ${ }^{1}$ Department of Mathematics, \\ Manickpur Adarsha Vidyapith, Howrah-711309, West Bengal, India. \\ e-mail: ghoshjay_04@yahoo.com \\ ${ }^{2}$ Department of Pure Mathematics, \\ University of Calcutta, 35 Ballygunge Circular Road, Kolkata-700019, India. \\ e-mail: dmandal.cu@gmail.com \\ ${ }^{3}$ Department of Mathematics, \\ Uluberia College, Howrah-711315, West Bengal, India. \\ e-mail: mumpu_tapas5@yahoo.co.in
}

\begin{abstract}
Concept of soft equivalence relations (classes, mappings) are introduced using the notion of soft elements. Then we redefine the notion of soft group and soft ring in a new way by using the idea of soft elements and it is seen that our definitions of soft group and soft ring are equivalent to the existing notions of soft group [2] and soft ring [1]. The notion of soft coset is presented and validated by suitable examples. We investigate some important properties like soft divisor of zero, characteristic of a soft ring etc. by considering examples. Moreover, some necessary and sufficient conditions are established for a soft ring to be a soft integral domain and soft field.
\end{abstract}

Key Words : Soft sets, Soft elements, Soft groups, Soft cosets, Soft rings, Soft integral domains, Soft fields.

2010 Mathematics Subject Classification: 06D72, 97E60, 97H40.

\section{Introduction}

In classical mathematics, we deal with precise or exact data. But almost all real life situations are imprecise or inexact, i.e., there are some ambiguity. As a result different mathematical tools such as probability theory, fuzzy set theory [15], rough set theory [13], soft set theory[11] etc. are developed to handle this imprecise situation. Soft set theory, introduced by D. Molodtsov

\footnotetext{
${ }^{1}$ Corresponding author
} 
in 1999, is more generalized tool than fuzzy set theory, rough set theory etc. After introduction of soft set theory, many researchers have contributed their works to extend this notion. In 2007, Aktas and Cagman[2] initiated an algebraic structure in a soft set, known as soft group. Extending this notion of soft group, many authors defined other algebraic structures like soft (ring, field, ideal) and hybrid structures like fuzzy soft (ring, field, ideal), intuitionistic fuzzy soft structures, rough soft structures (see [1], 4], [6]-9]) etc. In 2013, Wardowski[14] introduced the notion of soft elements of a soft set. Also in the same year, Nazmul and Samanta[12] defined soft elements in another way to study the neighbourhood properties in a soft topological spaces.

In this paper, at first we establish the notion of soft elements of a soft set in a new way and redefine the notion of soft relations, soft classes, soft partition, soft mapping with the help of soft element. It is seen that soft equivalence relation on a soft set yields a soft partition of that soft set and vice-versa. In section 4, the notion soft group is proposed with the help of binary composition of soft elements and study some of its properties. Then we prove that this notion is equivalent to the notion of soft group initiated by Aktas et al. In section 5, the notion of soft cosets are studied elaborately and it is established that the analogous result of famous Lagrange's Theorem holds for a finite soft group. In section 6 , we redefine the notion of soft ring by considering the compositions of soft elements. Then we prove that this

new concept of soft ring is also equivalent to the notion of soft ring given by Acar et al. [1]. Moreover, we study some properties like soft divisor of zero and characteristic of a soft ring with examples. In the last Section, some necessary and sufficient conditions are established for a soft ring to be a soft integral domain (soft field).

\section{Preliminaries}

Throughout this paper unless otherwise stated, let $U$ be the universal set, $E$ the set of all parameters with respect to $U$ and $P(U)$ the power set of $U$. In this section, we recall some basic definitions in soft set theory which will be needed in the sequel.

Definition 2.1. [1] Let $A \subseteq E$. A pair $(F, A)$ is called a soft set over $U$, where $F$ is a mapping given by $F: A \rightarrow P(U)$. Sometimes the soft set $(F, A)$ is simply denoted by $F$, when no confusion regarding the parameter set arises. The collection of all soft sets with parameter set $A$ over $U$ will be denoted by $S(A, U)$.

Definition 2.2. [1] Let $F, G \in S(A, U)$. Then $F$ is called a soft subset of $G$, denoted by $F \subseteq G$, if $F(t) \subseteq G(t)$ for all $t \in A$. 
Definition 2.3. [10] Let $F, G \in S(A, U)$. Then $F$ is soft equal to $G$, denoted by $F \cong G$, if $F \widetilde{\subseteq} G$ and $G \widetilde{\subseteq} F$.

Definition 2.4. 310 Let $F$ and $G \in S(A, U)$.

(1) $F \widetilde{\cap} G$, the intersection of $F$ and $G$, is defined by the soft set $H \in$ $S(A, U)$, where $H(t)=F(t) \cap G(t)$ for all $t \in A$.

(2) $F \widetilde{\cup} G$, the union of $F$ and $G$, is defined by the soft set $H \in S(A, U)$, where $H(t)=F(t) \cup G(t)$ for all $t \in A$.

(3) $F^{c}$, the complement of $F$, is defined by $F^{c}: A \rightarrow P(U)$, where $F^{c}(t)=$ $U \backslash F(t)$ for all $t \in A$.

(4) $F \backslash G$, the difference of $F$ and $G$, is defined by the soft set $H \in$ $S(A, U)$, where $H(t)=F(t) \backslash G(t)$ for all $t \in A$.

Definition 2.5. For $F \in S(A, U)$,

(i) $\operatorname{Supp}(F)=\{t \in A: F(t) \neq \phi\}$ is called support of the soft set $F$;

(ii) a soft set $F$ is said to be null soft set, denoted by $\Phi$, if $F(t)=\phi$ for all $t \in A$, otherwise $F$ is called non-null soft set;

(iii) a soft set $F$ is said to be absolute soft set, denoted by $\widetilde{A}$, if $F(t)=U$ for all $t \in A$;

(iv) a soft set $F$ is said to be full soft set if $\operatorname{Supp}(F)=A$.

The collection of all full soft sets with parameter set $A$ over $U$ will be denoted by $S_{f}(A, U)$.

Definition 2.6. [5] The soft cartesian product of $F$ and $G$, denoted by $F \widetilde{\times} G$, is defined as the soft set $F \widetilde{\times} G: A \times A \rightarrow P(U \times U)$, where $(F \widetilde{\times} G)\left(t_{1}, t_{2}\right)=F\left(t_{1}\right) \times G\left(t_{2}\right)$ for all $\left(t_{1}, t_{2}\right) \in A \times A$.

Definition 2.7. [5] A relation from $F$ to $G$ is a soft subset of $F \widetilde{\times} G$. In other words, a relation from $F$ to $G$ is of the form $\left(H_{1}, S\right)$ where $S \subseteq A \times A$ and $H_{1}\left(t_{1}, t_{2}\right)=F\left(t_{1}\right) \times G\left(t_{2}\right)$ for all $\left(t_{1}, t_{2}\right) \in S$.

\section{Soft relations and soft functions}

In this section, let $A \subseteq E$ and $F, G \in S_{f}(A, U)$. We initiate this section by establishing the notion of soft elements of a soft set.

Definition 3.1. A pair $(f, A)$ is called a soft element of $F$ if $f(t) \in$ $F(t)$ for all $t \in A$, where $f$ is a mapping given by $f: A \rightarrow U$. We simply write $f$ for the soft element $(f, A)$. The fact that $f$ is a soft element of $F$ will be denoted by $f \widetilde{\in} F$.

Note 3.2. For the existence of a soft element, we must have to take $F(t) \neq \phi$ for all $t \in A$, i.e., $F$ must be a full soft set. 
Definition 3.3. (1) A soft set $F$ is said to be a singleton soft set if $F(t)$ is a singleton set for each $t \in A$. In this case, $F$ is denoted simply by $\{f\}$, where $f$ is the only soft element of $F$.

(2) Two soft elements $f, g \widetilde{\in} F$ are said to be soft equal, denoted by $f \cong g$, if $f(t)=g(t)$ for all $t \in A$.

Note 3.4. A soft set $F \in S_{f}(A, U)$ may also be described by characterizing property of its soft elements, which is given by

$$
F=\{f: f: A \rightarrow U, \text { where } f(t) \in F(t), \forall t \in A\} .
$$

Some properties of soft elements are listed in the following proposition.

Proposition 3.5. Let $F, G \in S_{f}(A, U)$. Then

(i) $f \widetilde{\in} F \Leftrightarrow\{f\} \widetilde{\subseteq} F$;

(ii) $F \widetilde{\subseteq} G \Leftrightarrow f \widetilde{\in} F$ implies $f \widetilde{\in} G$;

(iii) If $F \widetilde{\cap} G \in S_{f}(A, U)$ then $f \widetilde{\in} F \widetilde{\cap} G \Leftrightarrow f \widetilde{\in} F$ and $f \widetilde{\in} G$;

(iv) $f \widetilde{\in} F$ or $f \widetilde{\in} G \Rightarrow f \widetilde{\in} F \widetilde{\cup} G$;

(v) $f \widetilde{\in} F^{c} \Rightarrow f \widetilde{\notin} F$;

(vi) $f \widetilde{\in} F \backslash G \Rightarrow f \widetilde{\in} F$ and $f \widetilde{\notin} G$;

(vii) $F \cong \bigcup_{f \widetilde{\in} F}\{f\}$.

Example 3.6. Let $U=\left\{u_{1}, u_{2}, u_{3}, u_{4}\right\}, E=\left\{p_{1}, p_{2}, p_{3}\right\}$ and $A=$ $\left\{p_{1}, p_{2}\right\}$. Let $F: A \rightarrow P(U)$ is given by $F\left(p_{1}\right)=\left\{u_{1}, u_{2}\right\}, F\left(p_{2}\right)=$ $\left\{u_{2}, u_{3}\right\}$, and $G: A \rightarrow P(U)$ is given by $G\left(p_{1}\right)=\left\{u_{1}, u_{4}\right\}, G\left(p_{2}\right)=$ $\left\{u_{3}, u_{4}\right\}$. Hence we have $(F \widetilde{\cap} G)\left(p_{1}\right)=\left\{u_{1}\right\},(F \widetilde{\cap} G)\left(p_{2}\right)=\left\{u_{3}\right\}$ and $\left.(F \widetilde{\cup} G)\left(p_{1}\right)=\left\{u_{1}, u_{2}, u_{4}\right\},(F \widetilde{\cup} G)\left(p_{2}\right)=\left\{u_{2}, u_{3}, u_{4}\right\}\right)$.

Also $F^{c}\left(p_{1}\right)=\left\{u_{3}, u_{4}\right\}, F^{c}\left(p_{2}\right)=\left\{u_{1}, u_{4}\right\}$ and $(F \backslash G)\left(p_{1}\right)=\left\{u_{2}\right\}$, $(F \backslash G)\left(p_{2}\right)=\left\{u_{2}\right\}$.

Then the soft elements of $F$ are $f_{1} \cong\left\{\left(p_{1}, u_{1}\right),\left(p_{2}, u_{2}\right)\right\}$;

$f_{2} \cong\left\{\left(p_{1}, u_{1}\right),\left(p_{2}, u_{3}\right)\right\} ; f_{3} \cong\left\{\left(p_{1}, u_{2}\right),\left(p_{2}, u_{2}\right)\right\} ; f_{4} \cong\left\{\left(p_{1}, u_{2}\right),\left(p_{2}, u_{3}\right)\right\}$.

Also the soft elements of $G$ are $g_{1} \cong\left\{\left(p_{1}, u_{1}\right),\left(p_{2}, u_{3}\right)\right\}$;

$g_{2} \cong\left\{\left(p_{1}, u_{1}\right),\left(p_{2}, u_{4}\right)\right\} ; g_{3} \cong\left\{\left(p_{1}, u_{4}\right),\left(p_{2}, u_{3}\right)\right\} ; g_{4} \cong\left\{\left(p_{1}, u_{4}\right),\left(p_{2}, u_{4}\right)\right\}$.

So, the soft elements of $F \widetilde{\cup} G$ are $a_{1} \cong\left\{\left(p_{1}, u_{1}\right),\left(p_{2}, u_{2}\right)\right\}$;

$a_{2} \cong\left\{\left(p_{1}, u_{1}\right),\left(p_{2}, u_{3}\right)\right\} ; a_{3} \cong\left\{\left(p_{1}, u_{1}\right),\left(p_{2}, u_{4}\right)\right\} ; a_{4} \cong\left\{\left(p_{1}, u_{2}\right),\left(p_{2}, u_{2}\right)\right\} ;$

$a_{5} \cong\left\{\left(p_{1}, u_{2}\right),\left(p_{2}, u_{3}\right)\right\} ; a_{6} \cong\left\{\left(p_{1}, u_{2}\right),\left(p_{2}, u_{4}\right)\right\} ; a_{7} \cong\left\{\left(p_{1}, u_{4}\right),\left(p_{2}, u_{2}\right)\right\} ;$

$a_{8} \cong\left\{\left(p_{1}, u_{4}\right),\left(p_{2}, u_{3}\right)\right\} ; a_{9} \cong\left\{\left(p_{1}, u_{4}\right),\left(p_{2}, u_{4}\right)\right\}$.

Also the soft element of $F \backslash G$ is $b_{1} \cong\left\{\left(p_{1}, u_{2}\right),\left(p_{2}, u_{2}\right)\right\}$.

Remark 3.7. Converse parts of (iv)-(vi) in the Proposition 3.5 are not true in general. Continuing Example 3.6, it is seen that $a_{6} \cong\left\{\left(p_{1}, u_{2}\right),\left(p_{2}, u_{4}\right)\right\}$ is a soft element of $F \widetilde{\cup} G$ but $a_{6} \widetilde{\notin} F$ and $a_{6} \widetilde{\notin} G$.

Again $a_{3} \cong\left\{\left(p_{1}, u_{1}\right),\left(p_{2}, u_{4}\right)\right\}$ is not a soft element of $F$ and $F^{c}$. Also $f_{1} \cong\left\{\left(p_{1}, u_{1}\right),\left(p_{2}, u_{2}\right)\right\}$ is a soft element of $F$ but $f_{1} \widetilde{\notin} G$ and $f_{1} \widetilde{\notin} F \backslash G$. 
Note 3.8. Since $F \widetilde{\times} G \in S_{f}(A \times A, U \times U)$, any soft element $a$ of $F \widetilde{\times} G$ is a mapping $a: A \times A \rightarrow U \times U$ such that $a\left(t_{1}, t_{2}\right) \in F\left(t_{1}\right) \times$ $G\left(t_{2}\right)$ for all $\left(t_{1}, t_{2}\right) \in A \times A$. Let $f \widetilde{\in} F, g \widetilde{\in} G$. Now consider an ordered pair $(f, g)$, defined by $(f, g): A \times A \rightarrow U \times U$, where $(f, g)\left(t_{1}, t_{2}\right)=$ $\left(f\left(t_{1}\right), g\left(t_{2}\right)\right)$ for all $\left(t_{1}, t_{2}\right) \in A \times A$. Since $f\left(t_{1}\right) \in F\left(t_{1}\right), g\left(t_{2}\right) \in G\left(t_{2}\right)$ then $(f, g)\left(t_{1}, t_{2}\right) \in F\left(t_{1}\right) \times G\left(t_{2}\right)$ for all $\left(t_{1}, t_{2}\right) \in A \times A$. Hence the ordered pair $(f, g)$ is a particular type of soft element of $F \widetilde{\times} G$.

Remark 3.9. If $a$ is a soft element of $F \widetilde{\times} G$ then $a$ may not be of the form $(f, g)$ for some $f \widetilde{\in} F, g \widetilde{\in} G$. This fact is justified by the following example.

Example 3.10. Let $F, G$ be two soft sets as defined in the Example 3.6. Then $(F \widetilde{\times} G)\left(p_{1}, p_{1}\right)=F\left(p_{1}\right) \times G\left(p_{1}\right)=\left\{u_{1}, u_{2}\right\} \times\left\{u_{1}, u_{4}\right\}=\left\{\left(u_{1}, u_{1}\right)\right.$, $\left.\left(u_{1}, u_{4}\right),\left(u_{2}, u_{1}\right),\left(u_{2}, u_{4}\right)\right\}$. Similarly,

$(F \widetilde{\times} G)\left(p_{1}, p_{2}\right)=\left\{u_{1}, u_{2}\right\} \times\left\{u_{3}, u_{4}\right\}=\left\{\left(u_{1}, u_{3}\right),\left(u_{1}, u_{4}\right),\left(u_{2}, u_{3}\right),\left(u_{2}, u_{4}\right)\right\} ;$

$(F \widetilde{\times} G)\left(p_{2}, p_{1}\right)=\left\{u_{2}, u_{3}\right\} \times\left\{u_{1}, u_{4}\right\}=\left\{\left(u_{2}, u_{1}\right),\left(u_{2}, u_{4}\right),\left(u_{3}, u_{1}\right),\left(u_{3}, u_{4}\right)\right\} ;$

$(F \widetilde{\times} G)\left(p_{2}, p_{2}\right)=\left\{u_{2}, u_{3}\right\} \times\left\{u_{3}, u_{4}\right\}=\left\{\left(u_{2}, u_{3}\right),\left(u_{2}, u_{4}\right),\left(u_{3}, u_{3}\right),\left(u_{3}, u_{4}\right)\right\}$.

Now consider a soft element $a: A \times A \rightarrow U \times U$ be defined as follows:

$a\left(p_{1}, p_{1}\right)=\left(u_{1}, u_{1}\right) ; a\left(p_{1}, p_{2}\right)=\left(u_{1}, u_{3}\right) ;$

$a\left(p_{2}, p_{1}\right)=\left(u_{2}, u_{1}\right) ; a\left(p_{2}, p_{2}\right)=\left(u_{2}, u_{4}\right)$.

But there does not exist any $f \widetilde{\in} F, g \widetilde{\in} G$ such that $a \cong(f, g)$. In fact, each of the soft sets $F, G$ contains 4 soft elements and therefore, the number of soft elements of the form $(f, g)$ in $F \widetilde{\times} G$ is 16 but the total number of soft elements in $F \widetilde{\times} G$ is $4^{4}$.

Notation 3.11. The set of all soft elements of $F \widetilde{\times} G$ of the form $(f, g)$, where $f \widetilde{\in} F, g \widetilde{\in} G$ is denoted by $(F, G)$.

Now, we redefine the notion of soft relation by modifying the definition of soft relation given by Babitha et al.[5] in 2010.

Definition 3.12. A soft relation $\widetilde{R}$ from $F$ to $G$ is a soft subset of $F \widetilde{\times} G$, i.e., $\widetilde{R}\left(t_{1}, t_{2}\right) \subseteq F\left(t_{1}\right) \times G\left(t_{2}\right)$ for all $\left(t_{1}, t_{2}\right) \in A \times A$. In particular, a soft relation on $F$ is a soft subset of $F \widetilde{\times} F$.

Note 3.13. Let $\widetilde{R}$ be a soft relation from $F$ to $G$. A soft element $f \widetilde{\in} F$ is related to $g \widetilde{\in} G$ by the soft relation $\widetilde{R}$ if $(f, g) \widetilde{\in} \widetilde{R}$ and it is expressed by $f \widetilde{R} g$. If $(f, g) \widetilde{\notin} \widetilde{R}$ then $f \widetilde{\in} F$ is not related to $g \widetilde{\in} G$ by the soft relation $\widetilde{R}$, which is denoted by $f \widetilde{R}^{\prime} g$.

Definition 3.14. Let $\widetilde{R}$ be a soft relation from $F$ to $G$. Then the domain of $\widetilde{R}$ is denoted by $\operatorname{Dom}(\widetilde{R})$ and defined by the soft set $D: A \rightarrow$ $P(U)$ such that for all $t_{1} \in A$,

$D\left(t_{1}\right)=\left\{x \in F\left(t_{1}\right):(x, y) \in \widetilde{R}\left(t_{1}, t_{2}\right)\right.$ for some $t_{2} \in A$ and $\left.y \in G\left(t_{2}\right)\right\}$. 
The Image of $\widetilde{R}$ is denoted by $\operatorname{Im}(\widetilde{R})$ and defined by the soft set $I: A \rightarrow$ $P(U)$ such that for all $t_{1} \in A$, $I\left(t_{1}\right)=\left\{y \in G\left(t_{1}\right):(x, y) \in \widetilde{R}\left(t_{2}, t_{1}\right)\right.$ for some $t_{2} \in A$ and $\left.x \in F\left(t_{2}\right)\right\}$.

Note 3.15. From the Definition 3.14, it is clear that $\operatorname{Dom}(\widetilde{R}) \widetilde{\subseteq} F$ and $\operatorname{Im}(\widetilde{R}) \widetilde{\subseteq} G$.

Example 3.16. Continuing Example 3.10 , consider a soft relation $\widetilde{R}$ from $F$ to $G$ as: $\widetilde{R}\left(p_{1}, p_{1}\right)=\left\{\left(u_{2}, u_{1}\right)\right\} ; \widetilde{R}\left(p_{1}, p_{2}\right)=\left\{\left(u_{2}, u_{4}\right)\right\} ; \widetilde{R}\left(p_{2}, p_{1}\right)=$ $\left\{\left(u_{3}, u_{1}\right)\right\} ; \widetilde{R}\left(p_{2}, p_{2}\right)=\left\{\left(u_{3}, u_{4}\right)\right\}$. Then $\operatorname{dom} \widetilde{R}\left(p_{1}\right)=\left\{u_{2}\right\}, \operatorname{dom} \widetilde{R}\left(p_{2}\right)=$ $\left\{u_{3}\right\}$ and $\operatorname{ran} \widetilde{R}\left(p_{1}\right)=\left\{u_{1}\right\}, \operatorname{ran} \widetilde{R}\left(p_{2}\right)=\left\{u_{4}\right\}$. From Example 3.6 and Note 3.13, it follows that $f_{4} \widetilde{R} g_{2}$ and $f_{4} \widetilde{R}^{\prime} g_{3}$.

Using the notion of soft elements the concepts of reflexive, symmetric, transitive and equivalence relations on a soft set are presented below:

Definition 3.17. Let $\widetilde{R}$ be a soft relation on $F$. Then

(1) $\widetilde{R}$ is said to be soft reflexive if $(f, f) \widetilde{\in} \widetilde{R}$ for all $f \widetilde{\in} F$.

(2) $\widetilde{R}$ is said to be soft symmetric if for any $f, g \widetilde{\in} F$,

$(f, g) \widetilde{\in} \widetilde{R} \Rightarrow(g, f) \widetilde{\in} \widetilde{R}$.

(3) $\widetilde{R}$ is said to be soft transitive if for any $f, g, h \widetilde{\in} F$,

$(f, g) \widetilde{\in} \widetilde{R}$ and $(g, h) \widetilde{\in} \widetilde{R} \Rightarrow(f, h) \widetilde{\in} \widetilde{R}$.

(4) $\widetilde{R}$ is said to be a soft equivalence relation if $\widetilde{R}$ is soft reflexive, soft symmetric and soft transitive.

Example 3.18. Consider the soft set $F \in S_{f}(A, U)$ as in the Example 3.6 and a soft relation $\widetilde{R}$ on $F$ as follows:

$\widetilde{R}\left(p_{1}, p_{1}\right)=\left\{\left(u_{1}, u_{1}\right),\left(u_{1}, u_{2}\right),\left(u_{2}, u_{2}\right)\right\}$;

$\widetilde{R}\left(p_{1}, p_{2}\right)=\left\{\left(u_{1}, u_{2}\right),\left(u_{1}, u_{3}\right),\left(u_{2}, u_{2}\right),\left(u_{2}, u_{3}\right)\right\} ;$

$\widetilde{R}\left(p_{2}, p_{1}\right)=\left\{\left(u_{2}, u_{1}\right),\left(u_{2}, u_{2}\right),\left(u_{3}, u_{1}\right),\left(u_{3}, u_{2}\right)\right\}$;

$\widetilde{R}\left(p_{2}, p_{2}\right)=\left\{\left(u_{2}, u_{2}\right),\left(u_{2}, u_{3}\right),\left(u_{3}, u_{3}\right)\right\}$.

The soft elements of $F$ are $f_{1} \cong\left\{\left(p_{1}, u_{1}\right),\left(p_{2}, u_{2}\right)\right\} ; f_{2} \cong\left\{\left(p_{1}, u_{1}\right),\left(p_{2}, u_{3}\right)\right\}$; $f_{3} \cong\left\{\left(p_{1}, u_{2}\right),\left(p_{2}, u_{2}\right)\right\} ; f_{4} \cong\left\{\left(p_{1}, u_{2}\right),\left(p_{2}, u_{3}\right)\right\}$. Now $\left(f_{1}, f_{1}\right)\left(p_{1}, p_{1}\right)=$ $\left(u_{1}, u_{1}\right) \in \widetilde{R}\left(p_{1}, p_{1}\right) ;\left(f_{1}, f_{1}\right)\left(p_{1}, p_{2}\right)=\left(u_{1}, u_{2}\right) \in \widetilde{R}\left(p_{1}, p_{2}\right) ;$

$\left(f_{1}, f_{1}\right)\left(p_{2}, p_{1}\right)=\left(u_{2}, u_{1}\right) \in \widetilde{R}\left(p_{2}, p_{1}\right) ;\left(f_{1}, f_{1}\right)\left(p_{2}, p_{2}\right)=\left(u_{2}, u_{2}\right) \in \widetilde{R}\left(p_{2}, p_{2}\right)$. Hence $\left(f_{1}, f_{1}\right) \widetilde{\in} \widetilde{R}$. Similarly, it follows that $\left(f_{2}, f_{2}\right),\left(f_{3}, f_{3}\right),\left(f_{4}, f_{4}\right) \widetilde{\in} \widetilde{R}$. So, $\widetilde{R}$ is a soft reflexive relation on $F$. Again, it also follows that $\left(f_{1}, f_{2}\right)$, $\left(f_{1}, f_{3}\right),\left(f_{1}, f_{4}\right),\left(f_{2}, f_{4}\right),\left(f_{3}, f_{4}\right) \widetilde{\in} \widetilde{R}$. It is seen that $\left(f_{1}, f_{2}\right) \widetilde{\in} \widetilde{R}$ but $\left(f_{2}, f_{1}\right) \widetilde{\notin} \widetilde{R}$. So, $\widetilde{R}$ is not a soft symmetric relation on $F$. Here $\widetilde{R}$ is a soft transitive relation on $F$.

Example 3.19. Consider the soft set $F \in S_{f}(A, U)$ as in the Example 3.6 and a soft relation $\widetilde{T}$ on $F$ as follows: 
$\widetilde{T}\left(p_{1}, p_{1}\right)=\left\{\left(u_{1}, u_{1}\right),\left(u_{1}, u_{2}\right),\left(u_{2}, u_{1}\right)\left(u_{2}, u_{2}\right)\right\}$

$\widetilde{T}\left(p_{1}, p_{2}\right)=\left\{\left(u_{1}, u_{2}\right),\left(u_{1}, u_{3}\right),\left(u_{2}, u_{2}\right),\left(u_{2}, u_{3}\right)\right\}$;

$\widetilde{T}\left(p_{2}, p_{1}\right)=\left\{\left(u_{2}, u_{1}\right),\left(u_{2}, u_{2}\right),\left(u_{3}, u_{1}\right),\left(u_{3}, u_{2}\right)\right\}$;

$\widetilde{T}\left(p_{2}, p_{2}\right)=\left\{\left(u_{2}, u_{2}\right),\left(u_{2}, u_{3}\right),\left(u_{3}, u_{2}\right),\left(u_{3}, u_{3}\right)\right\}$.

Then it can be verified that $\widetilde{T}$ is a soft reflexive, soft symmetric and soft transitive relation on $F$. Therefore $\widetilde{T}$ is a soft equivalence relation on $F$.

Definition 3.20. Let $F \in S_{f}(A, U)$ and $\widetilde{R}$ be a soft relation on $F$. (1) If $\widetilde{R} \cong F \widetilde{\times} F$ then $\widetilde{R}$ is called the universal soft relation on $F$. In this case $f \underset{\widetilde{R}}{\widetilde{R}} g$ hold for all $f, g \widetilde{\in} F$.

(2) If $\widetilde{R} \cong \Phi$, where $\Phi: A \times A \rightarrow P(U \times U)$ is a null soft set, then $\widetilde{R}$ is called the null soft relation on $F$. In this case $f \widetilde{R}^{\prime} g$ for all $f, g \widetilde{\in} F$.

Note 3.21. The universal soft relation on a soft set $F$ is a soft equivalence relation. Since the null soft relation is not soft reflexive, it can not be a soft equivalence relation.

Definition 3.22. Let $\widetilde{R}$ be a soft relation on $F$. The soft inverse of the soft relation $\widetilde{R}$, denoted by $\widetilde{R}^{-1}$, is defined by $\widetilde{R}^{-1}(t, s)=\widetilde{R}(s, t)$ for all $t, s \in A$.

Proposition 3.23. Let $\widetilde{R}$ be a soft relation on $F$. Then

(1) $\widetilde{R}$ is soft symmetric if and only if $(a, b) \in \widetilde{R}(t, s) \Rightarrow(b, a) \in \widetilde{R}(s, t)$ for all $t, s \in A$.

(2) $\widetilde{R}$ is soft symmetric if and only if $\widetilde{R}^{-1}$ is soft symmetric.

Proof. (1) Let $\widetilde{R}$ be a soft symmetric relation on $F$ and $(a, b) \in \widetilde{R}(t, s)$ for some $t, s \in A$. Then there exists at least one ordered pair $(f, g) \widetilde{\in} \widetilde{R}$ such that $(f, g)(t, s)=(a, b)$. This implies $f(t)=a, g(s)=b$. Since $\widetilde{R}$ is soft symmetric, by Definition 3.17, we have $(g, f) \widetilde{\in} \widetilde{R}$. Hence $(b, a)=$ $(g, f)(s, t) \in \widetilde{R}(s, t)$.

Conversely, let $(a, b) \in \widetilde{R}(t, s) \Rightarrow(b, a) \in \widetilde{R}(s, t) \forall t, s \in A$. Then $(f, g) \widetilde{\in} \widetilde{R}$ $\Rightarrow(f, g)(t, s) \in \widetilde{R}(t, s), \forall t, s \in A \Rightarrow(f(t), g(s)) \in \widetilde{R}(t, s), \forall t, s \in A \Rightarrow$ $(g(s), f(t)) \in \underset{R}{\widetilde{R}}(s, t), \forall t, s \in A \Rightarrow(g, f) \widetilde{\in} \widetilde{R}$. So, $\widetilde{R}$ is soft symmetric.

(2) Suppose $\widetilde{R}$ is soft symmetric. Then $(f, g) \widetilde{\in} \widetilde{R}^{-1} \Rightarrow(f, g)(t, s) \in$ $\widetilde{R}^{-1}(t, s), \forall t, s \in A \Rightarrow(f(t), g(s)) \in \widetilde{R}^{-1}(t, s)=\widetilde{R}(s, t), \forall t, s \in A \Rightarrow$ $(g(s), f(t)) \in \widetilde{R}(t, s), \forall t, s \in A, \Rightarrow(g(s), f(t)) \in \widetilde{R}^{-1}(s, t), \forall t, s \in A \Rightarrow$ $(g, f) \widetilde{\in} \widetilde{R}^{-1}$. Hence $\widetilde{R}^{-1}$ is soft symmetric. Converse part is similar.

Theorem 3.24. Let $\widetilde{S}, \widetilde{T}$ be two soft equivalence relation on $F$. Then $\widetilde{S} \widetilde{\cap} \widetilde{T}$ is also a soft equivalence relation on $F$.

Proof. From Proposition 3.5(iii) and Definition 3.17. Theorem follows. 
Next we confined ourself for the development of the notion of soft equivalence class relative to a soft equivalence relation on a soft set.

Definition 3.25. Let $\widetilde{R}$ be a soft equivalence relation on $F$. For all $f \widetilde{\in} F$, the soft equivalence class of $f$ is defined to be the soft set $L: A \rightarrow$ $P(U)$, where $L(t)=\underset{(g, f) \widetilde{\in} \widetilde{R}}{\widetilde{U}}\{g(t): g \widetilde{\in} F\}$ for all $t \in A$, and it can also be written as $L \cong \bigcup_{(g, f) \widetilde{\in} \widetilde{R}}^{\widetilde{N}}\{g: g \widetilde{\in} F\}$.

The soft equivalence class of $f$ will be denoted by $[f]_{\widetilde{R}}$ instead of $L$.

Note 3.26. Since $\widetilde{R}$ is soft reflexive, $(f, f) \widetilde{\in} \widetilde{R}$. Therefore $f \widetilde{\in}[f]_{\widetilde{R}}$.

Note 3.27. Since $g \widetilde{\in} F,\{g\} \widetilde{\simeq} F$. Therefore $[f]_{\widetilde{R}} \widetilde{\subseteq} F$.

In the following theorem, some basic properties of soft equivalence classes are presented.

Theorem 3.28. Let $\widetilde{R}$ be a soft equivalence relation on $F$. Then (i) for all $f \widetilde{\in} F,[f]_{\widetilde{R}} \approx \Phi$, where $\Phi$ is a null soft set,

(ii) for all $f, g \widetilde{\in} F,[f]_{\widetilde{R}} \cong[g]_{\widetilde{R}} \Leftrightarrow f \widetilde{R} g$,

(iii) for all $f, g \widetilde{\in} F$, if $f \widetilde{R}^{\prime} g$ then $[f]_{\widetilde{R}} \widetilde{\cap}[g]_{\widetilde{R}}$ contains no soft element of $F$, (iv) for all $f, g \widetilde{\in} F$, either $[f]_{\widetilde{R}} \cong[g]_{\widetilde{R}}$ or $[f]_{\widetilde{R}} \widetilde{\cap}[g]_{\widetilde{R}}$ contains no soft element of $F$.

(v) $F \cong \bigcup_{f \widetilde{\in} F}[f]_{\widetilde{R}}$.

Note 3.29. (1) If $f \widetilde{R}^{\prime} g$ then $[f]_{\widetilde{R}} \widetilde{\cap}[g]_{\widetilde{R}}$ may not be soft equal to $\Phi$. It is justified by the Example 3.37 .

(2) If two or more soft equivalence classes have no common soft element, then the soft equivalence classes are said to be distinct.

The definition of soft partition, given by Babitha and Sunil [5] is described by:

Definition 3.30. [5] Let $F \in S_{f}(A, U)$. A family $\left\{F_{\alpha}: \alpha \in I\right\}, I$ being the index set, of non-null soft subsets of $F$ is said to form a soft partition of $F$ if

(i) $F_{\alpha} \widetilde{\cap} F_{\beta} \cong \Phi$ for all $\alpha, \beta \in I, \alpha \neq \beta$ and

(ii) $F \cong \bigcup_{\alpha \in I} F_{\alpha}$.

Example 3.31. Consider the soft set $F$ as in the Example 3.6, i.e., $F\left(p_{1}\right)=\left\{u_{1}, u_{2}\right\}, F\left(p_{2}\right)=\left\{u_{2}, u_{3}\right\}$. Take a family of soft subsets $\left\{F_{1}, F_{2}\right\}$ of $F$, where $F_{1}\left(p_{1}\right)=\left\{u_{1}\right\}, F_{1}\left(p_{2}\right)=\left\{u_{2}\right\}$ and $F_{2}\left(p_{1}\right)=\left\{u_{2}\right\}, F_{2}\left(p_{2}\right)=$ 
$\left\{u_{3}\right\}$. Then $F \cong F_{1} \widetilde{\cup} F_{2}$ and $F_{1} \widetilde{\cap} F_{2} \cong \Phi$. Then by above Definition $\left\{F_{1}, F_{2}\right\}$ form a soft partition of $F$. It is to be noted that $f_{2} \cong\left\{\left(p_{1}, u_{1}\right),\left(p_{2}, u_{3}\right)\right\}$, $f_{3} \cong\left\{\left(p_{1}, u_{2}\right),\left(p_{2}, u_{2}\right)\right\}$ are soft elements of $F$ (as in the Example 3.6) but neither $f_{2}, f_{3}$ are the soft elements of $F_{1}$ nor the soft elements of $F_{2}$.

Thus a soft partition over a soft set, in the sense of Babitha and Sunil [5], is not being able to exhaust the collection of all soft elements of the soft set. So, we are now in a position to modify the definition of soft partition over a soft set, which is described in the following.

Definition 3.32. Let $F \in S_{f}(A, U)$. A family $\left\{F_{\alpha}: \alpha \in I\right\}, I$ being the index set, of non-null soft subsets of $F$ is said to form a soft partition of $F$ if

(i) $f \widetilde{\in} F \Rightarrow \exists \alpha \in I$ such that $f \widetilde{\in} F_{\alpha}$, and

(ii) for all $\alpha \neq \beta \in I, F_{\alpha} \widetilde{\cap} F_{\beta}$ contains no soft element of $F$.

Example 3.33. According to Definition 3.32, the family of soft subsets $\left\{F_{1}, F_{2}\right\}$ of $F$ (as in the Example 3.31) is not a soft partition of $F$. Continuing Example 3.31 , consider a family of soft subsets $\left\{F_{3}, F_{4}\right\}$ of $F$, where $F_{3}\left(p_{1}\right)=\left\{u_{1}\right\}, F_{3}\left(p_{2}\right)=\left\{u_{2}, u_{3}\right\}$ and $F_{4}\left(p_{1}\right)=\left\{u_{2}\right\}, F_{4}\left(p_{2}\right)=\left\{u_{2}, u_{3}\right\}$. Among the soft elements $f_{1} \cong\left\{\left(p_{1}, u_{1}\right),\left(p_{2}, u_{2}\right)\right\}$,

$f_{2} \cong\left\{\left(p_{1}, u_{1}\right),\left(p_{2}, u_{3}\right)\right\}, f_{3} \cong\left\{\left(p_{1}, u_{2}\right),\left(p_{2}, u_{2}\right)\right\}, f_{4} \cong\left\{\left(p_{1}, u_{2}\right),\left(p_{2}, u_{3}\right)\right\}$ of $F, f_{1}, f_{2} \widetilde{\in} F_{3}$ and $f_{3}, f_{4} \widetilde{\in} F_{4}$. Since $\left(F_{3} \widetilde{\cap} F_{4}\right)\left(p_{1}\right)=\phi,\left(F_{3} \widetilde{\cap} F_{4}\right)\left(p_{2}\right)=$ $\left\{u_{2}, u_{3}\right\}$, then $F_{3} \widetilde{\cap} F_{4}$ contains no soft element of $F$. By Definition 3.32 , $\left\{F_{3}, F_{4}\right\}$ form a soft partition of $F$.

Note 3.34. By condition (i) of Definition 3.32 it follows that $F \cong \bigcup_{\alpha \in I} F_{\alpha}$.

Note 3.35. For a soft equivalence relation $\widetilde{R}$ on $F$, if we consider the family $\mathcal{P}$ of all distinct soft equivalence classes, i.e., $\mathcal{P}=\left\{\left[f_{i}\right]_{\widetilde{R}}: f_{i} \widetilde{\in} F\right.$ and for $i \neq j,\left[f_{i}\right]_{\widetilde{R}} \widetilde{\cap}\left[f_{j}\right]_{\widetilde{R}}$ contains no soft element of $\left.F\right\}$. Then by Definition 3.32 , the family $\mathcal{P}$ of distinct soft equivalence classes constitutes a soft partition of the soft set $F$. Therefore each soft equivalence relation on a soft set $F$ yields a soft partition of $F$. In the next Theorem, the converse of this fact is proved.

Theorem 3.36. Let $\mathcal{P}=\left\{F_{\alpha}: \alpha \in I\right\}, I$ being the index set, be a soft partition of $F$. Then there exists a soft equivalence relation $\widetilde{R}$ on $F$ corresponding to the soft partition $\mathcal{P}$ such that every members of $\mathcal{P}$ become a soft equivalence class relative to the soft relation $\widetilde{R}$.

Proof. For any $f, g \widetilde{\in} F$, define a soft relation $\widetilde{R}$ on $F$ by $f \widetilde{R} g \Leftrightarrow f, g \widetilde{\in} F_{\alpha}$ for exactly one $F_{\alpha} \in \mathcal{P}$. Then it is similar task as crisp concept to prove that $\widetilde{R}$ is a soft equivalence relation on $F$. 
Now consider the soft equivalence class $[f]_{\widetilde{R}}$ for $f \widetilde{\in} F$. Since $f \widetilde{\in} F$, by definition of soft partition there exist $\alpha \in I$ such that $f \widetilde{\in} F_{\alpha}$. We claim that $[f]_{\widetilde{R}} \cong F_{\alpha}$. Since $g \widetilde{\in}[f]_{\widetilde{R}} \Leftrightarrow g \widetilde{R} f \Leftrightarrow g \widetilde{\in} F_{\alpha}$, therefore $[f]_{\widetilde{R}} \cong F_{\alpha}$. Conversely, if $F_{\alpha} \in \mathcal{P}, F_{\alpha} \stackrel{\cong}{=}[f]_{\widetilde{R}}$ for all $f \widetilde{\in} F_{\alpha}$.

Example 3.37. Let $U=\left\{u_{1}, u_{2}, u_{3}, u_{4}\right\}$ and $A=\left\{t_{1}, t_{2}\right\}$. Consider a soft set $F$, defined by $F\left(t_{1}\right)=\left\{u_{1}, u_{2}, u_{3}\right\}$ and $F\left(t_{2}\right)=\left\{u_{3}, u_{4}\right\}$. Then the soft elements of $F$ are $f_{1} \cong\left\{\left(t_{1}, u_{1}\right),\left(t_{2}, u_{3}\right)\right\}, f_{2} \cong\left\{\left(t_{1}, u_{1}\right),\left(t_{2}, u_{4}\right)\right\}$, $f_{3} \cong\left\{\left(t_{1}, u_{2}\right),\left(t_{2}, u_{3}\right)\right\}, f_{4} \cong\left\{\left(t_{1}, u_{2}\right),\left(t_{2}, u_{4}\right)\right\}, f_{5} \cong\left\{\left(t_{1}, u_{3}\right),\left(t_{2}, u_{3}\right)\right\}$, $f_{6} \cong\left\{\left(t_{1}, u_{3}\right),\left(t_{2}, u_{4}\right)\right\}$. Now consider a soft relation $\widetilde{R}$ on $F$ as follows: $\widetilde{R}\left(t_{1}, t_{1}\right)=\left\{\left(u_{1}, u_{1}\right),\left(u_{2}, u_{2}\right),\left(u_{1}, u_{2}\right),\left(u_{2}, u_{1}\right),\left(u_{3}, u_{3}\right)\right\} ;$ $\widetilde{R}\left(t_{1}, t_{2}\right)=\left\{\left(u_{1}, u_{3}\right),\left(u_{1}, u_{4}\right),\left(u_{2}, u_{3}\right),\left(u_{2}, u_{4}\right),\left(u_{3}, u_{3}\right),\left(u_{3}, u_{4}\right)\right\} ;$ $\widetilde{R}\left(t_{2}, t_{1}\right)=\left\{\left(u_{3}, u_{1}\right),\left(u_{4}, u_{1}\right),\left(u_{3}, u_{2}\right),\left(u_{4}, u_{2}\right),\left(u_{3}, u_{3}\right),\left(u_{4}, u_{3}\right)\right\} ;$ $\widetilde{R}\left(t_{2}, t_{2}\right)=\left\{\left(u_{3}, u_{3}\right),\left(u_{4}, u_{4}\right),\left(u_{3}, u_{4}\right),\left(u_{4}, u_{3}\right)\right\}$.

Then by Definition 3.17, $\widetilde{R}$ is a soft equivalence relation on $F$. Hence the distinct soft equivalence classes are $\left[f_{1}\right]_{\widetilde{R}} \cong\left\{f_{1}\right\} \widetilde{\cup}\left\{f_{2}\right\} \widetilde{\cup}\left\{f_{3}\right\} \widetilde{\cup}\left\{f_{4}\right\}$ and $\left[f_{5}\right]_{\widetilde{R}} \cong\left\{f_{5}\right\} \widetilde{\cup}\left\{f_{6}\right\}$. Therefore $\left\{\left[f_{1}\right]_{\widetilde{R}},\left[f_{5}\right]_{\widetilde{R}}\right\}$ forms a soft partition of $F$. Again $\left[f_{1}\right]_{\widetilde{R}}\left(t_{1}\right)=\left\{u_{1}, u_{2}\right\} ;\left[f_{1}\right]_{\widetilde{R}}\left(t_{2}\right)=\left\{u_{3}, u_{4}\right\}$ and $\left[f_{5}\right]_{\widetilde{R}}\left(t_{1}\right)=\left\{u_{3}\right\} ;$ $\left[f_{5}\right]_{\widetilde{R}}\left(t_{2}\right)=\left\{u_{3}, u_{4}\right\}$. Then $\left(\left[f_{1}\right]_{\widetilde{R}} \widetilde{\cap}\left[f_{5}\right]_{\widetilde{R}}\right)\left(t_{1}\right)=\phi$ and $\left(\left[f_{1}\right]_{\widetilde{R}} \widetilde{\cap}\left[f_{5}\right]_{\widetilde{R}}\right)\left(t_{2}\right)=$ $\left\{u_{3}, u_{4}\right\}$. So $\left[f_{1}\right]_{\widetilde{R}} \widetilde{\cap}\left[f_{5}\right]_{\widetilde{R}}$ is not a null soft set. On the other hand, we now consider a soft partition $\mathcal{P}=\left\{F_{1}, F_{2}\right\}$ of the above soft set $F$, where $F_{1}\left(t_{1}\right)=\left\{u_{1}, u_{2}\right\}, F_{1}\left(t_{2}\right)=\left\{u_{3}, u_{4}\right\}$ and $F_{2}\left(t_{1}\right)=\left\{u_{3}\right\}, F_{2}\left(t_{2}\right)=\left\{u_{3}, u_{4}\right\}$. For any $f, g \widetilde{\in} F$, define a soft relation $\widetilde{R}$ on $F$ by $f \widetilde{R} g \Leftrightarrow f, g \widetilde{\in} F_{i}$ for exactly one $F_{i} \in \mathcal{P}$. Then $\widetilde{R}$ is a soft equivalence relation on $F$ and $F_{1} \cong\left[f_{1}\right]_{\widetilde{R}} \cong\left[f_{2}\right]_{\widetilde{R}} \cong\left[f_{3}\right]_{\widetilde{R}} \cong\left[f_{4}\right]_{\widetilde{R}}, F_{2} \cong\left[f_{5}\right]_{\widetilde{R}} \cong\left[f_{6}\right]_{\widetilde{R}}$.

In the conclusion of this section, we now define soft function which will be needed in the subsequent section.

Definition 3.38. A soft relation $\widetilde{R}$ from $F$ to $G$ is said to be a soft function (or, soft mapping), denoted by $\widetilde{R}: F \rightarrow G$, if the following conditions are satisfied:

(1) $\operatorname{dom} \widetilde{R} \cong F$ and

(2) for each soft element $f \widetilde{\in} F$ there exist a unique soft element $g \widetilde{\in} G$

such that $f \widetilde{R} g$.

In this case $f \widetilde{R} g$ will be written as $\widetilde{R}(f) \cong g$.

Note 3.39. The set $\widetilde{R}(F)=\{\widetilde{R}(f): f \widetilde{\in} F\}$ is called the image set of $\widetilde{R}$ or the range set of $\widetilde{R}$. The image set $\widetilde{R}(F)$ is a soft subset of $G$.

Example 3.40. The soft relation $\widetilde{R}$ from $F$ to $G$, as defined in the Example 3.16, is not a soft function because $\operatorname{dom} \widetilde{R} \cong\left\{f_{4}\right\} \approx F$. 
Example 3.41. Let $F, G$ be two soft sets as described in the Example 3.6. Now define a soft relation $\widetilde{T}$ from $F$ to $G$ as follows: $\widetilde{T}\left(p_{1}, p_{1}\right)=\left\{\left(u_{1}, u_{1}\right),\left(u_{2}, u_{1}\right)\right\} ; \widetilde{T}\left(p_{1}, p_{2}\right)=\left\{\left(u_{1}, u_{4}\right),\left(u_{2}, u_{4}\right)\right\} ;$ $\widetilde{T}\left(p_{2}, p_{1}\right)=\left\{\left(u_{2}, u_{1}\right),\left(u_{3}, u_{1}\right)\right\} ; \widetilde{T}\left(p_{2}, p_{2}\right)=\left\{\left(u_{2}, u_{4}\right),\left(u_{3}, u_{4}\right)\right\}$. Then $\operatorname{dom} \widetilde{T}\left(p_{1}\right)=\left\{u_{1}, u_{2}\right\}, \operatorname{dom} \widetilde{T}\left(p_{2}\right)=\left\{u_{2}, u_{3}\right\}$. Hence $\operatorname{dom} \widetilde{T} \cong F$. Now it is easy to check that $\widetilde{T}\left(f_{1}\right) \cong g_{2} \cong \widetilde{T}\left(f_{2}\right) \cong \widetilde{T}\left(f_{3}\right) \cong \widetilde{T}\left(f_{4}\right)$. Therefore $\widetilde{T}$ is a soft mapping from $F$ to $G$ and $\widetilde{T}(F) \cong\left\{g_{2}\right\} \widetilde{\subseteq} G$.

Definition 3.42. (1) A soft mapping $\widetilde{R}: F \rightarrow G$ is said to be injective (one-one) if for any $f_{1}, f_{2} \widetilde{\in} F, f_{1} \underset{\neq}{\neq} f_{2} \Rightarrow \widetilde{R}\left(f_{1}\right) \widetilde{\neq} \widetilde{R}\left(f_{2}\right)$.

(2) A soft mapping $\widetilde{R}: F \rightarrow G$ is said to be surjective (onto) if $\widetilde{R}(F) \cong G$.

(3) A soft mapping $\widetilde{R}: F \rightarrow G$ is said to be bijective if $\widetilde{R}$ is both injective and surjective.

Example 3.43. The soft mapping given in the Example 3.41 is neither injective nor surjective. Now let $F, G$ be two soft sets and $f_{1}, f_{2}, f_{3}, f_{4}$ are the soft elements of $F$ and $g_{1}, g_{2}, g_{3}, g_{4}$ are the soft elements of $G$, as defined in the Example 3.6. Define a soft relation $\widetilde{Q}$ from $F$ to $G$ as follows:

$\widetilde{Q}\left(p_{1}, p_{1}\right)=\left\{\left(u_{1}, u_{1}\right),\left(u_{1}, u_{4}\right),\left(u_{2}, u_{1}\right),\left(u_{2}, u_{4}\right)\right\}$;

$\widetilde{Q}\left(p_{1}, p_{2}\right)=\left\{\left(u_{1}, u_{4}\right),\left(u_{2}, u_{3}\right)\right\}, \widetilde{Q}\left(p_{2}, p_{1}\right)=\left\{\left(u_{2}, u_{4}\right),\left(u_{3}, u_{1}\right)\right\}$, $\widetilde{Q}\left(p_{2}, p_{2}\right)=\left\{\left(u_{2}, u_{3}\right),\left(u_{2}, u_{4}\right),\left(u_{3}, u_{3}\right),\left(u_{3}, u_{4}\right)\right\}$.

Then by Definition 3.14. $\operatorname{dom} \widetilde{Q}\left(p_{1}\right)=\left\{u_{1}, u_{2}\right\}$, $\operatorname{dom} \widetilde{Q}\left(p_{2}\right)=\left\{u_{2}, u_{3}\right\}$. Hence $\operatorname{dom} \widetilde{Q} \cong F$ and $\widetilde{Q}\left(f_{1}\right) \cong g_{4}, \widetilde{Q}\left(f_{2}\right) \cong g_{2}, \widetilde{Q}\left(f_{3}\right) \cong g_{3}, \widetilde{Q}\left(f_{4}\right) \cong g_{1}$. Therefore $\widetilde{Q}$ is a soft mapping from $F$ to $G$ and also $\widetilde{Q}$ is bijective.

\section{Soft groups}

Throughout this section, let $(U, \circ)$ be a group and $F, G \in S_{f}(A, U)$. At first we confined ourself to introduce the notion of binary composition on a soft set. In crisp concept, a binary composition on a set $U$ is a mapping $f: U \times U \rightarrow U$, i.e., $f$ assigns to each ordered pair of elements of $U$ to a definite element of $U$. Since all soft elements of $F \widetilde{\times} F$ may not be of the form $(f, g)$ for some $f, g \widetilde{\in} F$, to define a binary composition on a soft set $F$, we restrict the domain of binary composition $F \widetilde{\times} F$ to $(F, F)$, the set of all soft elements of $F \widetilde{\times} F$ of the form $(f, g)$ for some $f, g \widetilde{\in} F$.

Definition 4.1. A binary composition on $F$ is a soft mapping $\widetilde{R}$ : $(F, F) \rightarrow F$. Then a binary composition on $F$ assigns to each ordered pair of soft elements of $F$ to a definite soft element of $F$. The binary 
composition $\widetilde{R}$ will be denoted by the symbols like $\widetilde{o}, \widetilde{*}$. The image of the soft element $(f, g)$ is denoted by the symbols like $f \widetilde{\circ} g, f \widetilde{*} g$.

Definition 4.2. Let $F \in S_{f}(A, U)$. Consider a binary composition $\widetilde{*}$ on $F$ as $(f \widetilde{*} g)(t)=f(t) \circ g(t)$ for all $t \in A$ and $f, g \widetilde{\in} F$. If $f \widetilde{*} g \widetilde{\in} F$ for all $f, g \widetilde{\in} F$ then the algebraic system $(F, \widetilde{*})$ is said to be a soft groupoid.

Note 4.3. If $F(t)$ forms a groupoid with respect to 'o' for all $t \in A$, then $(f \widetilde{*} g)(t)=f(t) \circ g(t) \in F(t)$ for all $t \in A$ and for $f, g \widetilde{\in} F$. Hence $f \widetilde{*} g \widetilde{\in} F$ for all $f, g \widetilde{\in} F$. Then $(F, \widetilde{*})$ forms a soft groupoid.

Definition 4.4. A soft groupoid $(F, \widetilde{*})$ is said to be a commutative soft groupoid (soft semigroup) if $\widetilde{*}$ is commutative (associative) on $F$ respectively.

Definition 4.5. (1) A soft element $e \widetilde{\in} F$ is said to be a soft identity element in a soft groupoid $(F, \widetilde{*})$ if $e \widetilde{*} f \cong f \widetilde{*} e \cong f$ for all $f \widetilde{\in} F$.

(2) In a soft groupoid $(F, \widetilde{*})$ with soft identity element $e$, a soft element $f \widetilde{\in} F$ is said to be invertible if there exist a soft element $f^{\prime} \widetilde{\in} F$ such that $f^{\prime} \tilde{*} f \cong f \widetilde{*} f^{\prime} \cong e$. Then $f^{\prime}$ is called a soft inverse of $f$.

In 2007, Aktas and Cagman [2] defined the notion of soft group as a parameterized family of subgroups of the universal group, which is as follows:

Definition 4.6. 2] $F$ is said to be a soft group over $U$ if $F(t)$ is a subgroup of $U$ for all $t \in A$.

Definition 4.7. [2] Let $F \subseteq \widetilde{\widetilde{C}} G$ and $G$ be a soft group over $U$. Then $F$ is said to be a soft subgroup of $G$, written as $F \widetilde{<} G$, if $F(t)$ is a subgroup of $G(t)$ for all $t \in A$.

We now redefine the notion of soft group as a binary composition of soft elements as follows:

Definition 4.8. A soft groupoid $(F, \widetilde{*})$ is said to be a soft group, where $\widetilde{*}$ is defined in the Definition 4.2 , if

(i) $\widetilde{*}$ is associative,

(ii) $\exists$ a soft element $e \widetilde{\in} F$ such that $e \widetilde{*} f \cong f \widetilde{*} e \cong f$ for all $f \widetilde{\in} F$,

(iii) for each soft element $f \widetilde{\in} F$, there exist a soft element $f^{\prime} \widetilde{\in} F$ such that $f^{\prime} \widetilde{*} \cong f \widetilde{*} f^{\prime} \cong e$.

Note 4.9. (1) The soft element $e \widetilde{\in} F$ is said to be a soft identity element in the soft group $(F, \widetilde{*})$. By condition (iii) of Definition 4.8, it follows that $e(t)=e_{U}$ (the identity element of $U$ ) for all $t \in A$. Therefore the soft identity element $e$ in a soft group is unique.

(2) The soft element $f^{\prime} \widetilde{\in} F$ is said to be a soft inverse of $f \widetilde{\in} F$. By condition (iv) of Definition 4.8 , it follows that $f^{\prime}(t)=[f(t)]^{-1}$ for all $t \in A$. Therefore each soft element $f \widetilde{\in} F$ has unique soft inverse. The soft inverse of a soft element $f$ will be denoted by $f^{-1}$. 
Definition 4.10. Let $F \widetilde{\subseteq} G$ and $(G, \widetilde{*})$ be a soft group. Then $(F, \widetilde{*})$ is said to be a soft subgroup of $(G, \widetilde{*})$ if $(F, \widetilde{*})$ forms a soft group.

Theorem 4.11. $(F, \widetilde{*})$ is a soft group if and only if $F(t)$ is a subgroup of $U$ for all $t \in A$.

Proof. Suppose $(F, \widetilde{*})$ forms a soft group. Let $t \in A$ be fixed but arbitrary. Let $a, b \in F(t)$. Then there exist $f, g \widetilde{\in} F$ such that $f(t)=a, g(t)=b$. Since $(F, \widetilde{*})$ is a soft group, $f \widetilde{*} g \widetilde{\in} F \Rightarrow a \circ b=f(t) \circ g(t)=(f \widetilde{*} g)(t) \in$ $F(t)$. $\circ$ is associative on $U$, since $\widetilde{*}$ is associative on $F$. By Note 4.9, $e(t)$ is the identity element of $F(t)$, where $e$ is the soft identity element of $F$. Now $f \widetilde{\in} F \Rightarrow f^{-1} \widetilde{\in} F$. Hence $a \circ f^{-1}(t)=f(t) \circ f^{-1}(t)=\left(f \widetilde{*} f^{-1}\right)(t)=e(t)$, the identity element of $F(t)$. Similarly, $f^{-1}(t) \circ a=e(t)$. Therefore each $a \in F(t)$ has an inverse in $F(t)$. Since $t \in A$ is arbitrary, $F(t)$ is a subgroup of $U$ for all $t \in A$.

Conversely, let $F(t)$ is a subgroup of $U$ for all $t \in A$. Then by Note 4.3, $(F, \widetilde{*})$ forms a soft groupoid. Clearly, $\widetilde{*}$ is associative on $F$. The soft element $e \widetilde{\in} F$, where $e(t)=e_{U}$ (identity element of $U$ ) for all $t \in A$, is the soft identity element of $F$. Let $f \widetilde{\in} F$. Then $f^{-1}(t)=[f(t)]^{-1} \in F(t)$ for all $t \in A \Rightarrow f^{-1} \widetilde{\in} F$. Therefore $(F, \widetilde{*})$ forms a soft group.

Remark 4.12. From the Theorem 4.11, it is seen that the notion of soft group given by Aktas et al. is equivalent to that of the notion given in the Definition 4.8. But this alternative definition of soft group gives us freedom to think a soft group like an ordinary group. Hence all the elementary group properties may hold for a soft group, which is a matter of investigation.

In the following theorems, some properties of a soft group (soft subgroup) are stated and the proofs are being omitted as these are straightforward.

Theorem 4.13. Let $F$ be a soft group over $U$. Then

(i) left and right cancellation law holds in $F$,

(ii) for all $f, g \widetilde{\in} F,(f \widetilde{*} g)^{-1} \cong g^{-1} \widetilde{*} f^{-1}$.

Theorem 4.14. Let $F \widetilde{\subseteq} G$ and $G$ be a soft group over $U$. Then $F \widetilde{<} G$ if and only if for all $f, g \widetilde{\in} F, f \widetilde{*} g^{-1} \widetilde{\in} F$.

Example 4.15. Let $U=S_{3}=\left\{\rho_{0}, \rho_{1}, \rho_{2}, \rho_{3}, \rho_{4}, \rho_{5}\right\}$, where $\rho_{0}=$ the identity permutation, $\rho_{1}=(1,2,3), \rho_{2}=(1,3,2), \rho_{3}=(2,3)$, $\rho_{4}=(1,3), \rho_{5}=(1,2)$. Then $(U, \circ)$ is a group, where ' $\circ$ ' is the usual composition of mappings. Consider the parameter set $A=\left\{t_{1}, t_{2}\right\}$ and define a soft set $F: A \rightarrow P(U)$ as $F\left(t_{1}\right)=\left\{\rho_{0}, \rho_{1}, \rho_{2}\right\}$ and $F\left(t_{2}\right)=\left\{\rho_{0}, \rho_{3}\right\}$. Since $F\left(t_{1}\right), F\left(t_{2}\right)$ are subgroups of $U$, by Definition 4.6, $F$ is a soft group over $U$. Now the soft elements of $F$ are $f_{1} \cong\left\{\left(t_{1}, \rho_{0}\right),\left(t_{2}, \rho_{0}\right)\right\}, f_{2} \cong\left\{\left(t_{1}, \rho_{0}\right),\left(t_{2}, \rho_{3}\right)\right\}$, 
$f_{3} \cong\left\{\left(t_{1}, \rho_{1}\right),\left(t_{2}, \rho_{0}\right)\right\}, f_{4} \cong\left\{\left(t_{1}, \rho_{1}\right),\left(t_{2}, \rho_{3}\right)\right\}, f_{5} \cong\left\{\left(t_{1}, \rho_{2}\right),\left(t_{2}, \rho_{0}\right)\right\}$, $f_{6} \cong\left\{\left(t_{1}, \rho_{2}\right),\left(t_{2}, \rho_{3}\right)\right\}$. Define a binary composition $\widetilde{*}$ on $F$ as in the Definition 4.2, i.e., $\left(f_{i} \widetilde{*} f_{j}\right)(t)=f_{i}(t) \circ f_{j}(t)$ for all $f_{i}, f_{j} \widetilde{\in} F$ and $t \in A$.

Then the composition table of $\widetilde{*}$ is given by:

\begin{tabular}{|c|c|c|c|c|c|c|}
\hline$\widetilde{*}$ & $f_{1}$ & $f_{2}$ & $f_{3}$ & $f_{4}$ & $f_{5}$ & $f_{6}$ \\
\hline$f_{1}$ & $f_{1}$ & $f_{2}$ & $f_{3}$ & $f_{4}$ & $f_{5}$ & $f_{6}$ \\
\hline$f_{2}$ & $f_{2}$ & $f_{1}$ & $f_{4}$ & $f_{3}$ & $f_{6}$ & $f_{5}$ \\
\hline$f_{3}$ & $f_{3}$ & $f_{4}$ & $f_{5}$ & $f_{6}$ & $f_{1}$ & $f_{2}$ \\
\hline$f_{4}$ & $f_{4}$ & $f_{3}$ & $f_{6}$ & $f_{5}$ & $f_{2}$ & $f_{1}$ \\
\hline$f_{5}$ & $f_{5}$ & $f_{6}$ & $f_{1}$ & $f_{2}$ & $f_{3}$ & $f_{4}$ \\
\hline$f_{6}$ & $f_{6}$ & $f_{5}$ & $f_{2}$ & $f_{1}$ & $f_{4}$ & $f_{3}$ \\
\hline
\end{tabular}

Hence $(F, \widetilde{*})$ forms a soft commutative group. The soft identity element is $f_{1}$ and $f_{2}^{-1} \cong f_{2}, f_{3}^{-1} \cong f_{5}, f_{4}^{-1} \cong f_{6}$. Then the soft subgroups of $F$ are given by $F_{0} \cong\left\{f_{1}\right\} ; F_{1} \cong\left\{f_{1}\right\} \widetilde{\cup}\left\{f_{2}\right\} ; F_{2} \cong\left\{f_{1}\right\} \widetilde{\cup}\left\{f_{3}\right\} \widetilde{\cup}\left\{f_{5}\right\}$ and $F$.

Definition 4.16. A soft group $F$ over $U$ is said to be a finite soft group if $F$ contains a finite number of soft elements. The order of a finite soft group $F$, denoted by $|F|$, is the number of soft elements of $F$.

Definition 4.17. Let $(F, \widetilde{*})$ be a soft group. For all positive integer $n$, define the integral power of a soft element $f \widetilde{\in} F$ by $f^{n} \cong f \widetilde{*} f \widetilde{*} \ldots \widetilde{*} f(n$ times $)$ i.e., $f^{n}(t)=f(t) \circ f(t) \circ \ldots \circ f(t), \forall t \in A$. Similarly, $f^{-n} \cong f^{-1} \widetilde{*} f^{-1} \widetilde{*} \ldots \widetilde{*} f^{-1}$ ( $n$ times $)$ and $f^{0} \cong e$, where $e$ is the soft identity element of $F$.

Definition 4.18. Let $(F, \widetilde{*})$ be a soft group. The order of a soft element $f \widetilde{\in} F$, denoted by $\circ(f)$, is the least positive integer $n$ such that $f^{n} \cong e$, where $e$ is the soft identity element of $F$. If such positive integer exist then $f$ is said to be of finite order otherwise $f$ is said to be of infinite order.

Example 4.19. As a continuation of Example 4.15, we see that $|F|=6$ and $\circ\left(f_{1}\right)=1, \circ\left(f_{2}\right)=2, \circ\left(f_{3}\right)=3, \circ\left(f_{4}\right)=6, \circ\left(f_{5}\right)=3, \circ\left(f_{6}\right)=6$.

\section{Soft cosets}

Throughout this section, $(U, \circ)$ is a group and $H, G \in S_{f}(A, U)$.

Definition 5.1. Let $G$ be a soft group over $U$ and $H \widetilde{<} G$. For any $a \widetilde{\in} G$, the soft set $a \widetilde{*} H: A \rightarrow P(U)$, defined by $(a \widetilde{*} H)(t)=a(t) \circ H(t)$ for all $t \in A$, is called a soft left coset of $H$ in $G$ determined by $a$. Similarly, the soft set $H \widetilde{*} a: A \rightarrow P(U)$, defined by $(H \widetilde{*} a)(t)=H(t) \circ a(t)$ for all $t \in A$, is called a soft right coset of $H$ in $G$ determined by $a$. 
Remark 5.2. Let $a \tilde{\in} G$. Then $f \widetilde{\in} a \widetilde{*} H \Rightarrow f(t) \in(a \widetilde{*} H)(t)=a(t) \circ$ $H(t)$ for all $t \in A$. In particular, if $A=\left\{t_{1}, t_{2}\right\}, f\left(t_{1}\right)=a\left(t_{1}\right) \circ u$, where $u \in H\left(t_{1}\right)$ and $f\left(t_{2}\right)=a\left(t_{2}\right) \circ v$, where $v \in H\left(t_{2}\right)$. From the Definition 3.1, there must exist a soft element $h \widetilde{\in} H$ such that $h\left(t_{1}\right)=u, h\left(t_{2}\right)=v$. Hence $f\left(t_{1}\right)=a\left(t_{1}\right) \circ h\left(t_{1}\right)=(a \tilde{*} h)\left(t_{1}\right)$ and $f\left(t_{2}\right)=a\left(t_{2}\right) \circ h\left(t_{2}\right)=(a \tilde{*} h)\left(t_{2}\right)$. Therefore $f \cong a \widetilde{*} h$ for some $h \widetilde{\in} H$. So, the soft left coset $a \widetilde{*} H$ may also be described as $a \widetilde{*} H \cong\{a \widetilde{*} h: h \widetilde{\in} H\}$. Similarly, the soft right coset $H \widetilde{*} a$ may be described as $H \widetilde{*} a \cong\{h \widetilde{*} a: h \widetilde{\in} H\}$.

Note 5.3. If $G$ is a soft group and $H \widetilde{<} G$, then $G(t)$ is a subgroup of $U$ and also $H(t)$ is a subgroup of $G(t)$ for all $t \in A$. Again, $a \widetilde{\in} G$ implies $a(t) \in G(t)$ for all $t \in A$. Therefore $(a \widetilde{*} H)(t)=a(t) \circ H(t) \subseteq G(t)$ for all $t \in A$. So, $a \widetilde{*} H \widetilde{\simeq} G$ for all $a \widetilde{\in} G$. Similarly, $H \widetilde{*} a \widetilde{\subseteq} G$ for all $a \widetilde{\in} G$.

Note 5.4. If $H \widetilde{<} G$, then the soft identity element $e \widetilde{\in} H$. For $a \tilde{\in} G$, $a(t)=a(t) \circ e(t) \in a(t) \circ H(t)$ for all $t \in A$. Therefore $a \tilde{\in} a \widetilde{*} H$. Similarly, $a \widetilde{\in} H \widetilde{*} a$.

Example 5.5. As a continuation of Example 4.15, consider the soft $\operatorname{group}(F, \widetilde{*})$ and the soft $\operatorname{subgroup}\left(F_{1}, \widetilde{*}\right)$, where $F_{1} \cong\left\{f_{1}\right\} \widetilde{\cup}\left\{f_{2}\right\}$. Then soft left cosets of $F_{1}$ in $F$ are $f_{1} \widetilde{*} F_{1} \cong\left\{f_{1}\right\} \widetilde{\cup}\left\{f_{2}\right\} \cong F_{1} ; f_{2} \widetilde{*} F_{1} \cong F_{1}$; $f_{3} \widetilde{*} F_{1} \cong\left\{f_{3}\right\} \widetilde{\cup}\left\{f_{4}\right\} \cong f_{4} \widetilde{*} F_{1} ; f_{5} \widetilde{*} F_{1} \cong\left\{f_{5}\right\} \widetilde{\cup}\left\{f_{6}\right\} \cong f_{6} \widetilde{*} F_{1}$. Hence $F_{1}\left(t_{1}\right)=$ $\left\{\rho_{0}\right\}, F_{1}\left(t_{2}\right)=\left\{\rho_{0}, \rho_{3}\right\} ;\left(f_{3} \widetilde{*} F_{1}\right)\left(t_{1}\right)=\left\{\rho_{1}\right\},\left(f_{3} \widetilde{*} F_{1}\right)\left(t_{2}\right)=\left\{\rho_{0}, \rho_{3}\right\}$ and $\left(f_{5} \widetilde{*} F_{1}\right)\left(t_{1}\right)=\left\{\rho_{2}\right\},\left(f_{5} \widetilde{*} F_{1}\right)\left(t_{2}\right)=\left\{\rho_{0}, \rho_{3}\right\}$.

Theorem 5.6. Let $G$ be a soft group over $U$ and $H \widetilde{<} G$. Then $h \widetilde{\in} H$ if and only if $h \widetilde{*} H \cong H$.

Proof. To prove $h \widetilde{*} H \cong H$, we have to prove $(h \widetilde{*} H)(t)=H(t)$ for all $t \in$ $A$. Let $t \in A$ be fixed. Since $G$ be a soft group over $U$ and $H \widetilde{<} G$, then $(G(t), \circ)$ is a group and $H(t)$ is a subgroup of $G(t)$. Moreover, $h \widetilde{\in} H \Rightarrow$ $h(t) \in H(t)$. Since $h(t) \circ H(t)$ is a left coset of $H(t)$ in $G(t), h(t) \circ H(t)=$ $H(t)$. This implies $(h \widetilde{*} H)(t)=H(t)$. Since $t \in A$ is arbitrary, $h \widetilde{*} H \cong H$. Conversely, let $h \widetilde{*} H \cong H$. By Note 5.4, $h \widetilde{\in} h \widetilde{*} H \Rightarrow h \widetilde{\in} H$.

Theorem 5.7. Let $G$ be a soft group over $U$ and $H \widetilde{<} G$. Then $a \widetilde{\in} G \backslash H$ if and only if $(a \widetilde{*} H) \widetilde{\cap} H \cong \Phi$.

Proof. From the given condition, we have $(G(t), \circ)$ is a group and $H(t)$ is a subgroup of $G(t)$ for all $t \in A$. Now $a \widetilde{\in} G \backslash H \Leftrightarrow a(t) \in G(t) \backslash H(t), \forall t \in$ $A \Leftrightarrow[a(t) \circ H(t)] \cap H(t)=\phi, \forall t \in A \Leftrightarrow(a \widetilde{*} H) \widetilde{\cap} H \cong \Phi$.

Theorem 5.8. Let $G$ be a soft group over $U$ and $H \widetilde{<} G$. Then $a \widetilde{\in} G, a \widetilde{\notin} H \Leftrightarrow(a \widetilde{*} H) \widetilde{\cap} H$ contains no soft element of $G$. 
Proof. At first let $a \widetilde{\in} G, a \widetilde{\notin} H$. Suppose $(a \widetilde{*} H) \widetilde{\cap} H$ contains a soft element $f$. Then $f \widetilde{\in}(a \widetilde{*} H) \widetilde{\cap} H \Rightarrow f \widetilde{\in} a \widetilde{*} H$ and $f \widetilde{\in} H \Rightarrow \exists h \widetilde{\in} H$ such that $f \cong a \widetilde{*} h \Rightarrow a \cong f \widetilde{*} h^{-1} \widetilde{\in} H$ (since $H \widetilde{<} G$ ). This is a contradiction. Therefore $(a \widetilde{*} H) \widetilde{\cap} H$ contains no soft element of $G$.

Conversely, let $(a \widetilde{*} H) \widetilde{\cap} H$ contains no soft element of $G$. By Note 5.4 . $a \widetilde{\in} a \widetilde{*} H$. If $a \widetilde{\in} H$, then $a \widetilde{\in}(a \widetilde{*} H) \widetilde{\cap} H$. It contradicts our assumption. Therefore $a \tilde{\notin} H$.

Theorem 5.9. Let $G$ be a soft group over $U$ and $H \widetilde{<} G$. For any $a, b \widetilde{\in} G$, either $a \widetilde{*} H \cong b \widetilde{*} H$ or $(a \widetilde{*} H) \widetilde{\cap}(b \widetilde{*} H)$ contains no soft element of $G$.

Proof. Suppose $(a \tilde{*} H) \widetilde{\cap}(b \widetilde{*} H)$ contains a soft element, say $f$. Then $f \widetilde{\in}(a \widetilde{*} H) \widetilde{\cap}(b \widetilde{*} H) \Rightarrow f(t) \in[a(t) \circ H(t)] \cap[b(t) \circ H(t)] \neq \phi, \forall t \in A$.

Since for all $t \in A, a(t), b(t) \in G(t), H(t)<G(t)$ and $a(t) \circ H(t), b(t) \circ$ $H(t)$ are left cosets of $H(t)$ in $G(t)$, then by crisp concept for $t \in A$, $[a(t) \circ H(t)] \cap[b(t) \circ H(t)] \neq \phi \Rightarrow a(t) \circ H(t)=b(t) \circ H(t)$. This implies $a \widetilde{*} H \cong b \widetilde{*} H$.

Example 5.10. In this example, we will show that there exist $a, b \widetilde{\in} G$ such that $a \widetilde{*} H \not b \widetilde{*} H$ and $(a \widetilde{*} H) \widetilde{\cap}(b \widetilde{*} H) \approx \Phi$. From Example 5.5, we see that $\left(f_{3} \widetilde{*} F_{1}\right) \widetilde{\cap}\left(f_{5} \widetilde{*} F_{1}\right) \cong\left\{\left(t_{1}, \phi\right),\left(t_{2},\left\{\rho_{0}, \rho_{3}\right\}\right)\right\} \approx \Phi$. Also $f_{3} \widetilde{*} F_{1} \nsucceq$ $f_{5} \widetilde{*} F_{1}$.

Theorem 5.11. Let $G$ be a soft group over $U$ and $H \widetilde{<} G$. The soft relation $\widetilde{R}$ on $G$ defined by $f \widetilde{R} g \Leftrightarrow f^{-1} \widetilde{*} g \widetilde{\in} H, \forall f, g \widetilde{\in} G$ is a soft equivalence relation on $G$ and each equivalence class is a soft left coset of $H$ in $G$, i.e., for all $f \widetilde{\in} G,[f]_{\widetilde{R}} \cong f \widetilde{*} H$.

Note 5.12. Since each equivalence class is a soft left coset of $H$ in $G$, then by Note 3.35, $G$ is partitioned into distinct soft left cosets.

Theorem 5.13. Let $G$ be a soft group over $U$ and $H \widetilde{<} G$. Then the soft elements of $H$ are in one-one correspondence with the soft elements of any soft left coset of $H$ in $G$.

Proof. Let $a \widetilde{\in} G$ and $a \widetilde{*} H$ be a soft left coset of $H$ in $G$. Let us define a soft mapping $\widetilde{R}: H \rightarrow a \widetilde{*} H$ by $\widetilde{R}(h) \cong a \widetilde{*} h$ for all $h \widetilde{\in} H$. Suppose $h_{1}, h_{2} \widetilde{\in} H$. Then $\widetilde{R}\left(h_{1}\right) \cong \widetilde{R}\left(h_{2}\right) \Leftrightarrow a \widetilde{*} h_{1} \cong a \widetilde{*} h_{2} \Leftrightarrow h_{1} \cong h_{2}$ (by left cancellation law). Therefore $\widetilde{R}$ is well defined and injective. To show $\widetilde{R}$ is onto, let $g \widetilde{\in} a \widetilde{*} H$. Then by Remark 5.2, there exist $h \widetilde{\in} H$ such that $g \cong a \widetilde{*} h$. This implies $g \cong \widetilde{R}(h)$. Hence $R$ is surjective. Therefore $\widetilde{R}$ is bijective.

Corollary 5.14. Let $G$ be a soft group over $U$ and $H \widetilde{<} G$. Then for all $a \widetilde{\in} G,|H|=|a \widetilde{*} H|$. 
Definition 5.15. Let $G$ be a soft group over $U$ and $H \widetilde{<} G$. Then the number of distinct soft left (right) cosets of $H$ in $G$ is called the index of $H$ in $G$. The index of $H$ in $G$ is denoted by $[G: H]$.

In group theory, Lagrange's Theorem states that the order of every subgroups of a finite group divides the order of that finite group. The analogous result for a soft group is given in the following theorem.

Theorem 5.16. Let $G$ be a finite soft group over $U$ and $H \widetilde{<} G$. Then order of $H$ divides the order of $G$. Moreover, $|G|=[G: H]|H|$.

Proof. By the Note 5.12 and the Corollary 5.14, the proof follows.

Example 5.17. From Example 4.15, we have $\left|F_{0}\right|=1,\left|F_{1}\right|=2,\left|F_{2}\right|=$ 3 and $|F|=6$.

\section{Soft rings}

Throughout this section, let $(U,+,$.$) be a ring and F \in S_{f}(A, U)$. We initiate this section by rewriting the notion of soft ring as a parameterized family of subrings of the universal ring, which was introduced by Acar et al.[1] in 2010 .

Definition 6.1. [1] $F$ is called a soft ring over $U$ if $F(t)$ is a subring of $U$ for all $t \in A$.

Definition 6.2. [1] Let $F \subseteq G$ and $G$ be a soft ring over $U$. Then $F$ is called a soft subring of $G$ if $F(t)$ is a subring of $G(t)$ for all $t \in A$.

Now we redefine the notion of a soft ring by using the binary compositions of its soft elements as follows:

Definition 6.3. $F$ is said to form a soft ring with respect to two binary compositions $\widetilde{+}$ and ? defined on $F$ for all $f, g \widetilde{\in} F$ as

$$
(f \widetilde{+} g)(t)=f(t)+g(t) \text { and }(f \sim g)(t)=f(t) \cdot g(t) \text { for all } t \in A
$$

if the following conditions are satisfied:

(i) $(F, \widetilde{+})$ is a commutative soft group;

(ii) $(F, \sim)$ is a soft semigroup and

(iii) for any three soft elements $f, g, h \widetilde{\in} F$,

the left distributive law $f^{\sim} \cdot(\underset{\widetilde{+}}{g} h) \cong f^{\sim} \cdot g \widetilde{+} f^{\sim} \cdot h$ and

the right distributive law $(g \widetilde{+} h) \sim f \cong g \sim f \widetilde{+} h \sim f$ both hold.

The soft ring is denoted by $(F, \widetilde{+}, \sim$ ) or simply by $F$ when no confusion regarding the underlying binary compositions arises. 
Theorem 6.4. ( $F, \widetilde{+}, \widetilde{r})$ is a soft ring if and only if $F(t)$ is a subring of $(U,+,$.$) for all t \in A$.

Proof. Suppose $(F, \tilde{+}, \tilde{.})$ is a soft ring. Then $(F, \tilde{+})$ is a commutative soft group, $(F, \stackrel{\sim}{.})$ is a soft semigroup and both distributive laws hold in $F$. Then by Theorem 4.11, $F(t)$ is a subgroup of $(U,+)$ for all $t \in A$ and $(F(t),$.$) is a semigroup for all t \in A$. Let $t \in A$ be fixed but arbitrary. Let $a, b, c \in F(t)$. Then there exist $f, g, h \widetilde{\in} F$ such that $f(t)=$ $a, g(t)=b, h(t)=c$. Hence $a+b=f(t)+g(t)=(f \widetilde{+} g)(t)=(g \tilde{+} f)(t)=$ $g(t)+f(t)=b+a$. So, $F(t)$ is a commutative subgroup of $(U,+)$. Again, $a \cdot(b+c)=f(t) \cdot[g(t)+h(t)]=f(t) \cdot(g \tilde{+} h)(t)=[f \sim(g \tilde{+} h)](t)=$ $[f \sim g \widetilde{+} f \sim \cdot h](t)=(f \sim g)(t)+(f \sim h)(t)=f(t) \cdot g(t)+f(t) \cdot h(t)=a \cdot b+a \cdot c$. So, the left distribution law holds in $F(t)$. Similarly, the right distribution law holds in $F(t)$. Since $t \in A$ is arbitrary, $F(t)$ is a subring of $(U,+,$. for all $t \in A$.

Conversely, suppose $F(t)$ is a subring of $(U,+,$.$) for all t \in A$. Then by Theorem 4.11, $(F, \widetilde{+})$ is a soft group and $(F, \sim)$ is a soft semigroup. Again $\widetilde{+}$ is commutative on $F$ as + is commutative on $U$. Also both the distribution laws hold in $(F, \widetilde{+}, \tilde{r})$ as the distributions of . over + hold in $F(t)$ for all $t \in A$. Therefore $(F, \widetilde{+}, \sim)$ forms a soft ring.

Remark 6.5. In the Theorem 6.4, it is established that the two notions of a soft ring given by Acar et al. and in the Definition 6.3 are equivalent. But this alternative definition of a soft ring allow us to think a soft ring as an ordinary ring. So, almost all the basic properties of an ordinary ring might be true for a soft ring. Hence we shall concentrate our aim to investigate such properties of a soft ring and note the differences from an ordinary ring.

Note 6.6. (1) The soft identity element in $F$ with respect to $\widetilde{+}$ is called the zero soft element in $F$. The zero soft element is unique and it is denoted by $0_{F}$. Here $0_{F}(t)=0$ for all $t \in A$, where 0 is the zero element of the ring $(U,+,$.$) . All other soft element in F$ except $0_{F}$ are called nonzero soft element in $F$.

(2) The soft inverse of a soft element $f \widetilde{\in} F$ with respect to $\widetilde{+}$ is called the additive soft inverse of $f$ and it is denoted by $-f$. Here $(-f)(t)=-f(t)$ for all $t \in A$, where $-f(t)$ is the additive inverse of $f(t)$ in the ground ring $(U,+,$.$) .$

Definition 6.7. (1) A soft element $e \widetilde{\in} F$ is said to be a multiplicative soft identity (or, soft unity) in $F$ if $e \widetilde{r} f \cong f^{\sim} \cdot e \cong f$ for all $f \widetilde{\in} F$.

(2) In a soft $\operatorname{ring}(F, \widetilde{+}, \sim)$ with soft unity $e$, a soft element $f \widetilde{\in} F$ is said to be a soft unit if there exists a soft element $g \widetilde{\in} F$ such that $f \sim g \cong g \sim f \cong e$. The soft element $g \widetilde{\in} F$ is said to be a multiplicative soft inverse of $f$.

(3) A soft ring $(F, \widetilde{+}, \sim)$ is said to be a commutative soft ring if $f \sim g \cong g \sim f$ for all $f, g \widetilde{\in} F$. 
Note 6.8. (1) By the Definition 6.7, the soft unity element in $F$ is unique and is denoted by $e$. Then it follows that $e(t) \cdot f(t)=f(t) \cdot e(t)=f(t)$ for all $t \in A, f \widetilde{\in} F$. Hence for a fixed $t \in A, e(t) . f(t)=f(t) \cdot e(t)=f(t)$ for all $f \widetilde{\in} F$. So, $e(t)$ is the unity element of the subring $F(t)$ for each $t \in A$. Since the subring $F(t)$ and the ground ring $(U,+,$.$) may have different$ unity elements, $e(t)$ may not be 1 , where 1 is the unity element of the $\operatorname{ring}(U,+,$.$) .$

(2) By the definition of multiplicative soft inverse, it follows that if $g \widetilde{\in} F$ is a multiplicative soft inverse of $f \widetilde{\in} F$ then $g(t)=[f(t)]^{-1}$ for all $t \in A$, where $[f(t)]^{-1}$ is the multiplicative inverse of $f(t)$ in the subring $F(t)$ for each $t \in A$. However, if a soft element $f \widetilde{\in} F$ has a multiplicative soft inverse in $F$ then the multiplicative soft inverse is unique and it is denoted by $f^{-1}$.

Note 6.9. In soft set theory, by trivial soft $\operatorname{ring} F$ we mean $F(t)=$ $\{0\} \forall t \in A$, i.e., $F$ contains only zero soft element and it is denoted by $\left(\left\{0_{F}\right\}, \tilde{+}, \tilde{r}\right)$. A non-trivial soft ring $F$ contains at least two soft elements.

Example 6.10. Let $U=\left(Z_{6},+,.\right)$ and $A=\left\{t_{1}, t_{2}\right\}$ be the parameter set. Consider a soft set $F: A \rightarrow P(U)$, defined as $F\left(t_{1}\right)=\{\overline{0}, \overline{3}\}$ and $F\left(t_{2}\right)=\{\overline{0}, \overline{2}, \overline{4}\}$. Then by the Theorem 6.4 . $(F, \widetilde{+}, \widetilde{r})$ is a soft ring, where $\widetilde{+}$, . are defined on $F$ as in the Equation (1). Here $\overline{3}, \overline{4}$ are the unities of the subrings $F\left(t_{1}\right), F\left(t_{2}\right)$ respectively. Then the soft unity element $e$ is given by $e \cong\left\{\left(t_{1}, \overline{3}\right),\left(t_{2}, \overline{4}\right)\right\}$. The soft elements $f \cong\left\{\left(t_{1}, \overline{3}\right),\left(t_{2}, \overline{2}\right)\right\}, g \cong$ $\left\{\left(t_{1}, \overline{3}\right),\left(t_{2}, \overline{4}\right)\right\}$ are the soft units in $F$ and both are multiplicative soft inverses of themselves. Here the soft ring $F$ is commutative.

Theorem 6.11. In a non-trivial soft ring with unity $e, 0_{F} \tilde{\neq} e$.

Definition 6.12. In a soft ring $F$, a non-zero soft element $f$ is said to be a soft divisor of zero if there exists a non-zero soft element $g \widetilde{\in} F$ such that either $f \widetilde{\sim} g \cong 0_{F}$ or $g \simeq f \cong 0_{F}$.

Example 6.13. Let $U=(Z,+,$.$) be the ring of all integers. Let$ $A=\left\{t_{1}, t_{2}\right\}$ be the parameter set. Consider a soft set $F: A \rightarrow P(U)$, defined as $F\left(t_{1}\right)=(2 Z,+,$.$) and F\left(t_{2}\right)=(3 Z,+,$.$) . Then by the The-$ orem 6.4. $(F, \widetilde{+}, \sim)$ is a soft ring, where $\tilde{+}, \sim$ are defined on $F$ as in the Equation (1) in Definition 6.3. Now consider two non-zero soft elements $f \cong\left\{\left(t_{1}, 0\right),\left(t_{2}, 3\right)\right\}, g \cong\left\{\left(t_{1}, 2\right),\left(t_{2}, 0\right)\right\} \widetilde{\in} F$. Then $(f \sim g)\left(t_{1}\right)=$ $f\left(t_{1}\right) \cdot g\left(t_{1}\right)=0$ and $(f \sim g)\left(t_{2}\right)=0$. Hence $f \sim g \cong 0_{F}$. So, $f, g$ are soft divisors of zero in $F$.

Theorem 6.14. A soft $\operatorname{ring}(F, \widetilde{+}, \sim)$ contains soft divisors of zero if and only if one of the following conditions hold:

(1) If $A$ contains only one parameter, $F(t)$ contains divisors of zero; 
(2) If $A$ contains more than one parameter, at least one subring, say $F\left(t_{i}\right)$, contains divisors of zero or, at least two subrings, say $F\left(t_{i}\right), F\left(t_{j}\right)$, are nontrivial, where $t_{i}, t_{j} \in A$.

Proof. Suppose the soft ring $(F, \tilde{+}, \tilde{.})$ contains soft divisors of zero. Then there exist non-zero soft elements $f, g \widetilde{\in} F$ such that $f \sim g \cong 0_{F}$. Now $f, g \neq 0_{F} \Rightarrow \exists t_{i}, t_{j} \in A$ such that $f\left(t_{i}\right) \neq 0$ and $g\left(t_{j}\right) \neq 0$. If $t_{i}=t_{j}$ then $f \sim g \cong 0_{F} \Rightarrow f\left(t_{i}\right) \cdot g\left(t_{i}\right)=0 \Rightarrow F\left(t_{i}\right)$ contains divisors of zero. If $t_{i} \neq t_{j}$ then the soft rings $F\left(t_{i}\right), F\left(t_{j}\right)$ are non-trivial. Hence the required conditions hold.

Conversely, since $(F, \widetilde{+}, \sim)$ is a soft ring then $F(t)$ is a subring of $(U,+,$. for all $t \in A$. Then $0 \in F(t)$ for all $t \in A$, where 0 is the zero element of $(U,+,$.$) . Let the parameter set A$ contains only one element, say $t$. Also suppose the subring $F(t)$ contains a divisor of zero, say $u(\neq 0)$. Then there exists another $v(\neq 0) \in F(t)$ such that either $u . v=0$ or $v \cdot u=0$. Now we can find $f, g \widetilde{\in} F$ such that $f(t)=u, g(t)=v$. Therefore $(f \sim g)(t)=f(t) \cdot g(t)=0$ or $(g \sim f)(t)=0$. So, either $f \sim g \cong 0_{F}$ or $g \simeq f \cong 0_{F}$. Therefore $f, g$ are soft divisors of zero in $F$.

Next, let the parameter set $A$ contains more than one element. If at least one subring, say $F\left(t_{i}\right)$ contains divisors of zero then there exist $u(\neq 0), v(\neq$ $0) \in F\left(t_{i}\right)$ such that $u \cdot v=0$ or $v \cdot u=0$. Consider two soft element $f, g \widetilde{\in} F$ such that $f\left(t_{i}\right)=u, f(t)=0$ for all $t\left(\neq t_{i}\right) \in A$ and $g\left(t_{i}\right)=$ $v, g(t)=0$ for all $t\left(\neq t_{i}\right) \in A$. Then either $f \simeq g \cong 0_{F}$ or $g \simeq f \cong 0_{F}$, where $f, g \neq 0_{F}$. Therefore $f, g$ are soft divisors of zero in $F$.

If at least two subrings, say $F\left(t_{i}\right), F\left(t_{j}\right)$, are non-trivial, we can find two soft elements $f, g \widetilde{\in} F$ such that $f\left(t_{i}\right)=u \neq 0, f(t)=0$ for all $t\left(\neq t_{i}\right) \in A$ and $g\left(t_{j}\right)=v \neq 0, g(t)=0$ for all $t\left(\neq t_{j}\right) \in A$. Then $f, g$ are non-zero soft elements of $F$ and $(f \sim g)(t)=0$ for all $t \in A$. This implies $f \sim g \cong 0_{F}$, the zero soft element of $F$. Therefore $f, g$ are soft divisors of zero in $F$.

Corollary 6.15. Let $(U,+,$.$) be a ring contains no divisor of zero and$ $F \in S_{f}(A, U)$. A soft ring $(F, \widetilde{+}, \tilde{r})$ contains soft divisors of zero if and only if $A$ contains more than one parameter and at least two subrings, say $F\left(t_{i}\right), F\left(t_{j}\right)$, are non-trivial.

Theorem 6.16. A non-trivial soft $\operatorname{ring}(F, \widetilde{+}, \sim)$ contains no soft divisor of zero if and only if for exactly one parameter $t_{i} \in A, F\left(t_{i}\right)$ is a non-trivial subring containing no divisor of zero and all other subring $F(t)$ becomes trivial, where $t\left(\neq t_{i}\right) \in A$.

Proof. Proof follows from the Theorem 6.14.

Note 6.17. In the Example 6.13, we have seen that the ring $(U,+,$. contains no divisor of zero, whereas the soft $\operatorname{ring}(F, \tilde{+}, \tilde{r})$ contains soft divisors of zero. 
Theorem 6.18. If $f$ be a soft unit in a soft $\operatorname{ring}(F, \widetilde{+}, \sim)$ with soft unity, then $f$ is not a soft divisor of zero.

Note 6.19. By Theorem 6.18, if $f$ is a soft divisor of zero in a soft ring $F$ then $f$ is not a soft unit in $F$. For a soft ring $F$, where at least two subrings among the subrings $F(t), t \in A$, are non-trivial, all the non-zero soft elements $f \widetilde{\in} F$, satisfying the condition $f\left(t_{i}\right)=0$ for at least one parameter $t_{i} \in A$ corresponding to the non-trivial subrings $F(t)$, are soft divisors of zero. Hence this type of soft elements are not soft unit in the above soft ring $F$. Suppose $A$ contains more than one parameter, say $n$ and $(F, A)$ is a non-trivial soft ring over $U$ such that $F\left(t_{1}\right), F\left(t_{2}\right)$ are non-trivial subring, where $t_{1}, t_{2} \in A$. Then the soft elements of the form $\left\{\left(t_{1}, 0\right),\left(t_{2}, u\right),\left(t_{3}, 0\right), \ldots,\left(t_{n}, 0\right)\right\}$ and $\left\{\left(t_{1}, v\right),\left(t_{2}, 0\right),\left(t_{3}, 0\right), \ldots,\left(t_{n}, 0\right)\right\}$, where $u, v(\neq 0) \in U$, are not soft units in $F$.

Definition 6.20. The characteristic of a soft $\operatorname{ring}(F, \widetilde{+}, \widetilde{r})$, denoted by char $F$, is the least positive integer $n$ such that $n f \cong 0_{F}$, where $n f \cong f \widetilde{+} f$ $\widetilde{+} \ldots \widetilde{+} f(n$ times $)$. If no such positive integer $n$ exists, $F$ is said to be of characteristic zero.

Note 6.21. By a finite soft ring $F$, we mean $F$ contains finite number of soft elements. Hence for a finite soft ring $F$, the corresponding parameter set $A$ and all the subrings $F(t)$, where $t \in A$, are finite.

Theorem 6.22. Characteristic of a finite soft $\operatorname{ring}(F, \widetilde{+}, \sim)$ is the l.c.m of the characteristics of the subrings $F(t)$ for all $t \in A$.

Proof. If the parameter set $A$ contains only one parameter then the theorem follows. Next, let the parameter set $A$ contains two elements, say $A=\left\{t_{1}, t_{2}\right\}$. Since $F$ is a finite soft ring then $F\left(t_{1}\right), F\left(t_{2}\right)$ are finite subrings and hence the characteristics of $F\left(t_{1}\right), F\left(t_{2}\right)$ are finite, say $m, n$ respectively. Let $\operatorname{lcm}(m, n)=r$. This implies $r f \cong 0_{F}$ for all $f \widetilde{\in} F$. Suppose $s$ be the positive integer less than $r$ such that $s f \cong 0_{F}$ for all $f \widetilde{\in} F$. Then $s f(t)=0$ for all $t \in A, f \widetilde{\in} F$. Since $m, n$ are the characteristics of the subrings $F\left(t_{1}\right)$ and $F\left(t_{2}\right)$ respectively, then both $m, n$ divides $s$. This implies $r$ divides $s$, which is a contradiction. So, $r$ is the least positive integer such that $r f \cong 0_{F}$ for all $f \widetilde{\in} F$. Therefore $\operatorname{char} F=\operatorname{lcm}(m, n)$. This theorem can be proved by considering another parameter set with more than two elements.

Note 6.23. Let $(F, \widetilde{+}, \sim)$ be a soft ring. Then $\operatorname{char} F=1$ if and only if $F$ is the trivial soft ring.

Example 6.24. In the Example 6.10, the characteristics of the subrings $F\left(t_{1}\right), F\left(t_{2}\right)$ are 2,3 respectively. It is easy to verify that the characteristic of the soft ring $F$ is 6 . 


\section{Soft integral domains and Soft fields}

Throughout this section unless otherwise stated, let $(U,+,$.$) be a ring and$ $F \in S_{f}(A, U)$.

Definition 7.1. A non-trivial soft $\operatorname{ring}(F, \widetilde{+}, \sim)$ with soft unity is said to be a soft integral domain if $F$ is commutative and contains no soft divisor of zero.

Theorem 7.2. A non-trivial commutative soft $\operatorname{ring}(F, \widetilde{+}, \sim)$ with soft unity is a soft integral domain if and only if for exactly one parameter $t_{i} \in A, F\left(t_{i}\right)$ becomes a non-trivial subring having no divisor of zero and all other $F(t)$ becomes a trivial subring, where $t\left(\neq t_{i}\right) \in A$.

Proof. The proof follows from the Theorem 6.16.

Note 7.3. (1) For a trivial ring, 0 is zero element as well as the unity element. Hence from the Note6.8, it follows that if $e$ is the soft unity element of a soft integral domain $F$ then $e\left(t_{i}\right)$ is the unity element of the non-trivial subring $F\left(t_{i}\right)$ and $e(t)=0$ for all $t\left(\neq t_{i}\right) \in A$, where 0 is the zero element of the ground ring $U$, i.e., soft unity element of a soft integral domain $F$ is of the form $\left\{\left(t_{1}, 0\right),\left(t_{2}, 0\right), \ldots,\left(t_{i-1}, 0\right),\left(t_{i}, u\right),\left(t_{i+1}, 0\right) \ldots,\left(t_{n}, 0\right)\right\}$, where $i, n \in \mathbb{N}$ and $u$ is the unity element of the non-trivial subring $F\left(t_{i}\right)$.

(2) If $f$ be a non-zero soft element of a soft integral domain $F$, then $f\left(t_{i}\right)$ is a non-zero element of the non-trivial subring $F\left(t_{i}\right)$ and $f(t)=0$ for all $t\left(\neq t_{i}\right) \in A$, i.e., each non-zero soft element of a soft integral domain $F$ is of the form $\left\{\left(t_{1}, 0\right),\left(t_{2}, 0\right), \ldots,\left(t_{i-1}, 0\right),\left(t_{i}, a\right),\left(t_{i+1}, 0\right) \ldots,\left(t_{n}, 0\right)\right\}$, where $i, n \in \mathbb{N}$ and $a(\neq 0) \in F\left(t_{i}\right)$.

(3) In a soft integral domain $F$, if there exist a multiplicative soft inverse of a non-zero soft element $\left\{\left(t_{1}, 0\right),\left(t_{2}, 0\right), \ldots,\left(t_{i-1}, 0\right),\left(t_{i}, a\right),\left(t_{i+1}, 0\right) \ldots,\left(t_{n}, 0\right)\right\}$, where $i, n \in \mathbb{N}$ and $a \neq 0$, then multiplicative soft inverse is of the form $\left\{\left(t_{1}, 0\right),\left(t_{2}, 0\right), \ldots,\left(t_{i-1}, 0\right),\left(t_{i}, a^{-1}\right),\left(t_{i+1}, 0\right) \ldots,\left(t_{n}, 0\right)\right\}$, where $i, n \in \mathbb{N}$ and $a^{-1}$ is the multiplicative inverse of $a$ in the non-trivial subring $F\left(t_{i}\right)$.

Example 7.4. 1. Let $U=\left(Z_{6},+,.\right)$ and $A=\left\{t_{1}, t_{2}\right\}$ be the parameter set. Consider a soft set $F: A \rightarrow P(U)$, defined as $F\left(t_{1}\right)=\{\overline{0}, \overline{2}, \overline{4}\}$ and $F\left(t_{2}\right)=\{\overline{0}\}$. Then by the Theorem 6.4. $(F, \widetilde{+}, \tilde{r})$ is a soft ring. Here $F$ is commutative and it contains the soft unity $\left\{\left(t_{1}, \overline{4}\right),\left(t_{2}, \overline{0}\right)\right\}$. Also $F$ contains no soft divisor of zero. So, $F$ is a soft integral domain.

2. The soft ring, as in the Example 6.10, is not a soft integral domain because it contains the soft divisors of zero $a \cong\left\{\left(t_{1}, \overline{3}\right),\left(t_{2}, \overline{0}\right)\right\}, b \cong\left\{\left(t_{1}, \overline{0}\right)\right.$, $\left.\left(t_{2}, \overline{2}\right)\right\}, c \cong\left\{\left(t_{1}, \overline{0}\right),\left(t_{2}, \overline{4}\right)\right\}$.

Corollary 7.5. A soft ring $(F, \widetilde{+}, \tilde{r})$ is a soft integral domain if and only if for exactly one parameter $t_{i} \in A, F\left(t_{i}\right)$ is an integral domain and all other $F(t)$ is a trivial subring, where $t\left(\neq t_{i}\right) \in A$. 
Corollary 7.6. Let $(U,+,$.$) be an integral domain. Then a soft ring$ $(F, \widetilde{+}, \sim)$ over $U$ is a soft integral domain if and only if for exactly one parameter $t_{i} \in A, F\left(t_{i}\right)$ is a nontrivial subring containing unity element and all other $F(t)$ is a trivial subring, where $t\left(\neq t_{i}\right) \in A$.

Theorem 7.7. The characteristic of a finite soft integral domain is a prime number.

Proof. Suppose $(F, \widetilde{+}, \sim)$ is a finite soft integral domain. If the parameter set $A$ contains only one parameter $t$, then by the Corollary $7.5, F(t)$ is a finite integral domain, whose characteristic is a prime number. Then by Definition 6.20, the characteristic of the soft integral domain $F$ is a prime number. If $A$ contain more than one parameter then by Corollary 7.5 , there exists only one parameter $t_{i} \in A$ such that $F\left(t_{i}\right)$ is a finite integral domain and all other $F(t)$ is a trivial subring, where $t\left(\neq t_{i}\right) \in A$. The characteristic of $F\left(t_{i}\right)$ is a prime number and the characteristic of $F(t)$ is 1 for all $t\left(\neq t_{i}\right) \in A$. Then by the Theorem 6.22 , the characteristic of $F$ is a prime number.

Definition 7.8. A non-trivial soft $\operatorname{ring}(F, \widetilde{+}, \sim)$ with soft unity is said to be a soft field if $F$ is commutative and each non-zero soft element of $F$ is a soft unit.

Example 7.9. In the Example 7.4, $F$ is a soft field, where the non-zero soft elements $\left\{\left(t_{1}, \overline{2}\right),\left(t_{2}, \overline{0}\right)\right\},\left\{\left(t_{1}, \overline{4}\right),\left(t_{2}, \overline{0}\right)\right\}$ are soft units.

Theorem 7.10. A soft field is a soft integral domain.

Proof. Suppose $(F, \tilde{+}, \sim$ ) be a soft field. Then each non-zero soft element of $F$ is a soft unit. By the Theorem 6.18, each non-zero soft element of $F$ is not a soft divisor of zero. Hence $F$ contains no soft divisor of zero and proving that $(F, \widetilde{+}, \widetilde{\sim})$ is a soft integral domain.

Note 7.11. Converse of the Theorem 7.10 is not true, i.e., a soft integral domain is not necessarily a soft field. It is justified by the following example.

Example 7.12. Let $U=(Q,+,$.$) be the ring of all rational num-$ bers. Let $A=\left\{t_{1}, t_{2}\right\}$ be the parameter set. Consider a soft set $F$ : $A \rightarrow P(U)$, defined as $F\left(t_{1}\right)=(Z,+,$.$) and F\left(t_{2}\right)=\{0\}$. Then by the Corollary 7.5, $(F, \widetilde{+}, \sim)$ is a soft integral domain. Each non-zero soft element $\left\{\left(t_{1}, u\right),\left(t_{2}, 0\right)\right\}$ of $F$ has no multiplicative soft inverse, because $u(\neq 0) \in F\left(t_{1}\right)$ having no multiplicative inverse in $F\left(t_{1}\right)$. Therefore $F$ is not a soft field.

Theorem 7.13. A non-trivial commutative soft $\operatorname{ring}(F, \widetilde{+}, \widetilde{r})$ with soft unity is a soft field if and only if for exactly one parameter $t_{i} \in A, F\left(t_{i}\right)$ is a non-trivial subring such that each non-zero element of $F\left(t_{i}\right)$ is a unit and all other $F(t)$ is a trivial subring, where $t\left(\neq t_{i}\right) \in A$. 
Proof. Suppose $(F, \tilde{+}, \sim)$ be a soft field. Then $F$ is a soft integral domain. So, by the Theorem 7.2 , there is exactly one parameter $t_{i} \in A$ such that $F\left(t_{i}\right)$ is a non-trivial subring having no divisor of zero and all other $F(t)$ is a trivial subring, where $t\left(\neq t_{i}\right) \in A$. Suppose $a(\neq 0) \in F\left(t_{i}\right)$. Then by Note 7.3, we can find a non-zero soft element in $F$ of the form $\left\{\left(t_{1}, 0\right),\left(t_{2}, 0\right), \ldots,\left(t_{i-1}, 0\right),\left(t_{i}, a\right),\left(t_{i+1}, 0\right) \ldots,\left(t_{n}, 0\right)\right\}$, where $i, n \in \mathbb{N}$ and $a(\neq 0) \in F\left(t_{i}\right)$ and the corresponding multiplicative soft inverse is of the form $\left\{\left(t_{1}, 0\right),\left(t_{2}, 0\right), \ldots,\left(t_{i-1}, 0\right),\left(t_{i}, a^{-1}\right),\left(t_{i+1}, 0\right) \ldots,\left(t_{n}, 0\right)\right\}$, where $i, n \in \mathbb{N}$ and $a^{-1}$ is the multiplicative inverse of $a$ in the non-trivial subring $F\left(t_{i}\right)$. Therefore each non-zero element of $F\left(t_{i}\right)$ is a unit.

Conversely, let $F\left(t_{i}\right)$ is a non-trivial subring such that each non-zero element of $F\left(t_{i}\right)$ is a unit and all other $F(t)$ is a trivial subring. Since a non-zero soft element $f \widetilde{\in} F$ is of the form $f\left(t_{i}\right)=a \neq 0$ and $f(t)=0$ for all $t\left(\neq t_{i}\right) \in A$. Then by Note 7.3, the non-zero soft element $g$ of the form $g\left(t_{i}\right)=a^{-1}$ and $g(t)=0$ for all $t\left(\neq t_{i}\right) \in A$ is the multiplicative soft inverse of $f$. Therefore $F$ is a soft field.

Corollary 7.14. A soft ring $(F, \widetilde{+}, \widetilde{r})$ is a soft field if and only if for exactly one parameter $t_{i} \in A, F\left(t_{i}\right)$ is a field and all other $F(t)$ is a trivial subring, where $t\left(\neq t_{i}\right) \in A$.

Theorem 7.15. A finite soft integral domain is a soft field.

Proof. Suppose $(F, \widetilde{+}, \sim)$ be a finite soft integral domain. By the Corollary 7.5. for exactly one parameter $t_{i} \in A, F\left(t_{i}\right)$ is a finite integral domain and all other $F(t)$ is a trivial subring, where $t\left(\neq t_{i}\right) \in A$. So, $F\left(t_{i}\right)$ is a field. Hence by the Corollary $7.14, F$ is a soft field.

\section{References}

[1] U. Acar, F. Koyuncu, B. Tanay, Soft sets and soft rings, Comput. Math. Appl. 59 ( 2010 ) 3458-3463.

[2] H. Aktas, N. Cagman, Soft sets and soft groups, Inform. Sci. 177 ( 2007 ) 2726-2735.

[3] M. I. Ali, F. Feng, X. Liu, W. K. Min, M. Shabir, On some new operations in soft set theory, Comput. Math. Appl. 57 ( 2009 ) 1547-1553.

[4] A. Aygunoglu, H. Aygun, Introduction to fuzzy soft groups, Comput. Math. Appl. 58 ( 2009 ) 1279-1286.

[5] K. V. Babitha, J. J. Sunil, Soft set relations and functions, Comput. Math. Appl. 60 ( 2010 ) 1840-1849. 
[6] F. Feng, Y. B. Jun, X. Zhao, Soft semirings, Comput. Math. Appl. 56 ( 2008 ) 2621-2628.

[7] F. Feng, C. Li, B. Davvaz, M. I. Ali, Soft sets combined with fuzzy sets and rough sets: a tentative approach, Soft Comput. 14 ( 2010 ) 899-911.

[8] J. Ghosh, B. Dinda, T. K. Samanta, Fuzzy soft rings and fuzzy soft ideals, Int. J. Pure Appl. Sci. Technol., 2 (2) (2011) 66-74.

[9] J. Ghosh, T. K. Samanta, Rough soft sets and rough soft groups, Journal of Hyperstructures 2 (1) ( 2013 ) 18-29..

[10] P. K. Maji, R. Biswas, A. R. Roy, Soft set theory, Comput Math Appl 45 ( 2003 ) 555-562.

[11] D. Molodtsov, Soft set theory-first results, Comput Math Appl 37 (1999) 19-31.

[12] Sk. Nazmul, S. K. Samanta, Neighbourhood properties of soft topological spaces, Ann. Fuzzy Math. Inform., 6 (1) (2013) 1-15.

[13] Z. Pawlak, Rough sets, Int. J. Inform. Comput. Sci. 11 ( 1982 ) 341-356.

[14] D. Wardowski, On a soft mapping and its fixed points, Fixed Point Theory and Applications 182 ( 2013 ) 1-11.

[15] L. Zadeh, Fuzzy sets, Inform. Control 8 ( 1965 ) 338-353. 